\title{
New approximations in local volatility models
}

\author{
E. Gobet and A. Suleiman
}

1 Ecole Polytechnique, CMAP, Route de Saclay, 91128 Palaiseau Cedex, FRANCE. Email: emmanuel.gobet@polytechnique.edu

2 Ensimag, 681 rue de la passerelle, Domaine Universitaire, 38402 St Martin d'Hères, FRANCE. Email: ali.suleiman9@gmail.com

\begin{abstract}
For general time-dependent local volatility models, we propose new approximation formulas for the price of call options. This extends previous results of [BGM10b] where stochastic expansions combined with Malliavin calculus were performed to obtain approximation formulas based on the local volatility At The Money. Here, we derive alternative expansions involving the local volatility at strike. Averaging both expansions give even more accurate results. Approximations of the implied volatility are provided as well.
\end{abstract}

Keywords. Local volatility model, European option, asymptotic expansion, CEV model.

The first author is grateful to Chair Financial Risks of the Risk Foundation for its financial support.

\subsection{Introduction}

\subsubsection{Framework}

We consider a linear Brownian motion $\left(W_{t}\right)_{0 \leq t \leq T}$ defined on a filtered probability space $\left(\Omega, \mathcal{F}_{T},\left(\mathcal{F}_{t}\right)_{0 \leq t \leq T}, \mathbb{P}\right)$ where $T>0$ is a fixed terminal time. Here, $\left(\mathcal{F}_{t}\right)_{0 \leq t \leq T}$ is the completion of the natural filtration of $W$. This is used to model the dynamics of a risky asset $S$ (e.g. a stock or an index), which price process is $\left(S_{t}\right)_{0 \leq t \leq T}$. We are mainly interested in valuing European-style financial contracts written on $S$, exercised at maturity $T$, which related payoff is of the form $\Phi\left(S_{T}\right)$. We especially pay attention to vanilla options, i.e. $\Phi(S)=(S-K)_{+}$(call options) and $\Phi(S)=(K-S)_{+}$(put options).

We consider the standard framework of complete market (see for instance [MR98]), and more specifically, we assume that

1. the short-term interest rate $\left(r_{t}\right)_{0 \leq t \leq T}$ is deterministic and bounded;

Page: 1 job:GOBET_SULEIMAN_VolLocale_FinalVersion macro: svmult.cls date/time: 5-0ct-2010/9:10 
2. the risky asset pays a continuous dividend $\left(q_{t}\right)_{0 \leq t \leq T}$, which is deterministic and bounded;

3. $S$ follows a local volatility model, which dynamics is defined by the solution of the following stochastic differential equation:

$$
\frac{d S_{t}}{S_{t}}=\left(r_{t}-q_{t}\right) d t+\sigma\left(t, S_{t}\right) d W_{t}
$$

We denote the compound factor by

$$
C_{t}=\exp \left(\int_{0}^{t}\left(r_{s}-q_{s}\right) d s\right)
$$

Thus, we have

$$
\begin{aligned}
S_{t} & =C_{t} \exp \left(X_{t}\right) \\
X_{t} & =\log \left(S_{0}\right)+\int_{0}^{t} \sigma\left(s, S_{s}\right) d W_{s}-\frac{1}{2} \int_{0}^{t} \sigma^{2}\left(s, S_{s}\right) d s .
\end{aligned}
$$

Note that the above dynamics are directly given under the risk-neutral measure, since we only focus on pricing formulas. Then, the option price at time 0 is given by $\mathbb{E}\left(e^{-\int_{0}^{T} r_{s} d s} \Phi\left(S_{T}\right)\right)$. Of course, due to the general form of the local volatility function $\sigma(t, S)$, it is hopeless to derive exact closed formulas for such option prices. The aim of this work is to obtain accurate approximations.

\subsubsection{Literature background}

The interest in local volatility models probably dates back to the work by Dupire [Dup94] among others, who shows that such models are able to fit all call and put option prices at a given observation date (the calibration date). However, except in a few cases, analytical pricing formulas are available (for instance, in the CEV model $\sigma(t, S)=\nu S^{\beta-1}$, see [Sch89]). As alternative numerical methods, one could use a PDE approach but to achieve real-time pricing and calibration routines, it is better to search for approximative formulas, quicker to evaluate. Hagan etal. [HW99] use singular perturbation techniques to obtain an implied volatility expansion, in the case of separable volatility $\sigma(t, S)=\alpha(t) A(S)$. Henry-Labordère [Hen08] transfers heat kernel expansions on price expansions. To tackle the case of non-separable volatility, Piterbarg [Pit05] suggests the use of parameter averaging for some choices of $\sigma(t, S)$. A different approach has been developed in [BGM09]: first a model proxy is chosen, then a smart expansion around this proxy is performed, involving Malliavin calculus to determine explicitly the expansion terms. This approach appears to be quite flexible since it naturally handles time-dependent coefficients and various modeling situations including so far jumps, discrete dividends or stochastic interest rates. More precisely, applications to local volatility model including jumps have been developed in [BGM09] and deeply

Page: 2 job:GOBET_SULEIMAN_VolLocale_FinalVersion macro: svmult.cls date/time: 5-Oct-2010/9:10 
investigated along further directions in [BGM10b]. Allowing the interest rates to be stochastic is achieved in [BGM10a], while [BGM10c] considers the case of time-dependent Heston model. In [EG10], the authors investigate the case of assets paying discrete dividends. Within this approach, we are able to prove explicit error estimates that depend on $\sigma$ and its derivatives, on the maturity and on the payoff. It helps to better understand the roles of each parameter. In addition, the regularity of the payoff is crucial in order to design the expansion and to establish error estimates. These features are extensively discussed in [BGM10b] and [BGM10a].

Nevertheless, regarding the results in [BGM10b], one could legitimately formulate the criticism that we use the local volatility only At The Money (ATM in short) when we take the model proxy as Black-Scholes model and when we compute the expansions. For arbitrary payoffs, this is natural, but for call/put options, this may be strange since the spot and strike variables play somewhat symmetric roles.

Here, we correct this drawback by providing new expansion formulas based on the local volatility at strike (and we even mix the expansions). This article is organized as follows: in the next paragraphs, we define the assumptions and notations used throughout the paper. Then, in the next section, main results are stated. The main proofs are postponed in Appendix. Numerical experiments are presented in Section 1.3.

\subsubsection{Standing assumptions for the approximations}

Throughout the paper, we assume the following:

- Assumption $(E)$. The function $\sigma$ is bounded and positive $\left(\sigma_{\text {inf }}=\right.$ $\left.\inf _{(t, x) \in[0, T] \times \mathbb{R}^{+}} \sigma(t, S)>0\right)$. We denote by $c_{E} \geq 1$ the smallest constant such that

$$
\sup _{(t, S) \in[0, T] \times \mathbb{R}^{+}} \sigma(t, S) \leq c_{E} \inf _{(t, S) \in[0, T] \times \mathbb{R}^{+}} \sigma(t, S) .
$$

- Assumption $(R)$. The function $\sigma$ is seven-times continuously differentiable in the $S$-variable and

$$
\begin{aligned}
& M_{1}=\max _{1 \leq i \leq 7} \sup _{(t, x) \in[0, T] \times \mathbb{R}}\left|\frac{\partial^{i}}{\partial x^{i}}[\sigma(t, \exp (x))]\right|<\infty, \\
& M_{0}=\max \left(M_{1}, \sup _{(t, S) \in[0, T] \times \mathbb{R}^{+}} \sigma(t, S)\right)<\infty .
\end{aligned}
$$

The assumption $(R)$ is used in at least two respects: it allows for differentiating coefficients to obtain an expansion formula; it is used to derive error estimates. The assumption $(E)$ is an ellipticity-type condition that enables us to handle the error analysis for non-smooth payoffs $\Phi$ (such as call/put options). This is the standard framework developed in [BGM09].

Note that for deterministic volatility functions, one has $M_{1}=0$.

Page: 3 job:GOBET_SULEIMAN_VolLocale_FinalVersion macro: svmult.cls date/time: 5-0ct-2010/9:10 


\subsubsection{Definitions and other notations}

In the representation of the expansion formulas, we repeatedly use the following integral operator.

Definition 1 (Integral Operator). The integral operator $\omega^{T}$ is defined as follows: for any integrable function $l$, we set

$$
\omega(l)_{t}^{T}=\int_{t}^{T} l_{u} d u
$$

for $t \in[0, T]$. Similarly, for integrable functions $\left(l_{1}, l_{2}\right)$, we put for $t \in[0, T]$

$$
\omega\left(l_{1}, l_{2}\right)_{t}^{T}=\omega\left(l_{1} \omega\left(l_{2}\right)_{.}^{T}\right)_{t}^{T}=\int_{t}^{T} l_{1, r}\left(\int_{r}^{T} l_{2, s} d s\right) d r .
$$

The n-times iteration is defined analogously: for any integrable functions $\left(l_{1}, \cdots, l_{n}\right)$, we set

$$
\omega\left(l_{1}, \cdots, l_{n}\right)_{t}^{T}=\omega\left(l_{1} \omega\left(l_{2}, \cdots, l_{n}\right)^{T}\right)_{t}^{T}
$$

for $t \in[0, T]$.

We also use a short notation for Greeks.

Definition 2 (Greeks). Let $Z$ be a random variable and let $h$ be a payoff function. We define the $i^{\text {th }}$ Greek for the variable $Z$ by the quantity (if it has a meaning):

$$
\operatorname{Greek}_{i}^{h}(Z)=\left.\frac{\partial^{i} \mathbb{E}[h(Z+x)]}{\partial x^{i}}\right|_{x=0} .
$$

Definition 3 (Black-Scholes formula and related Greeks). Using usual notation, the Black-Scholes formula for call option and constant parameters $(\sigma, r, q)$ writes

$$
\mathrm{Call}^{\mathrm{BS}}(t, S ; T, K ; \sigma, r, q)=S e^{-q(T-t)} \mathcal{N}\left(d_{1}\right)-K e^{-r(T-t)} \mathcal{N}\left(d_{2}\right),
$$

where $\mathcal{N}(d)=\int_{-\infty}^{d} \frac{e^{-u^{2} / 2}}{\sqrt{2 \pi}} d u$ and

$$
\begin{aligned}
& d_{1}=d_{1}(t, S ; T, K ; \sigma, r, q)=\frac{1}{\sigma \sqrt{T-t}} \log \left(\frac{S e^{-q(T-t)}}{K e^{-r(T-t)}}\right)+\frac{1}{2} \sigma \sqrt{T-t} \\
& d_{2}=d_{2}(t, S ; T, K ; \sigma, r, q)=d_{1}-\sigma \sqrt{T-t} .
\end{aligned}
$$

For time dependent coefficients $\left(\sigma_{s}, r_{s}, q_{s}\right)_{0 \leq s \leq T}$, the call price formula is deduced from the Black-Scholes formula by replacing the arguments $\sigma^{2}, r$ and $q$ by their time-average on the interval $[t, T]$. The resulting formula is denoted by $\mathrm{Call}^{\mathrm{BS}}\left(t, S ; T, K ;\left(\sigma_{s}\right)_{s},\left(r_{s}\right)_{s},\left(q_{s}\right)_{s}\right)$.

For $t<T$ and $\sigma>0$, the function $(S, K) \mapsto \operatorname{Call}^{\mathrm{BS}}(t, S ; T, K ; \sigma, r, q)$ is smooth and its sensitivities $\frac{\partial^{i}}{\partial S^{i}} \mathrm{Call}^{\mathrm{BS}}(t, S ; T, K ; \sigma, r, q)$ and $\frac{\partial^{i}}{\partial K^{i}} \mathrm{Call}^{\mathrm{BS}}(t, S ; T, K ; \sigma, r, q)$ are given explicitly in Proposition 1 (see Appendix $C$ ), for $i=1, \ldots, 6$. They will be used in our expansion formulas (see Theorems 2 and 3). 


\subsection{Expansion formulas}

In this section, we give several expansion formulas, with a second and third order accuracy. The general principle for deriving such approximations is to choose a relevant proxy and to expand the quantities of interest around this proxy. First, we recall the general results from [BGM09], where the proxy is obtained by freezing the local volatility at the initial spot value (ATM). Second we apply these expansions to call options. Third, using the Dupire forward PDE satisfied by the call price as a function of maturity and strike, we propose a new proxy where the volatility is frozen at the strike value $K$ (instead of $S_{0}$ ). We then derive new second and third approximation formulas. Finally, some expansions of implied volatility are provided.

\subsubsection{A general result}

We first state two expansion results in a quite general form, so that we can apply it later to various situations. Let $\left(Y_{t}\right)_{0 \leq t \leq T}$ the solution of

$$
d Y_{t}=-\frac{1}{2} a^{2}\left(t, Y_{t}\right) d t+a\left(t, Y_{t}\right) d W_{t}, \quad Y_{0} \text { given. }
$$

Theorem 1 (Second and third order approximations [BGM10b, Theorems 2.1 and 2.3]). Assume that

- the function $a$ is bounded and positive $\left(a_{\text {inf }}=\inf _{(t, y) \in[0, T] \times \mathbb{R}} a(t, y)>0\right)$. We denote by $c_{E} \geq 1$ the smallest constant such that

$$
\sup _{(t, y) \in[0, T] \times \mathbb{R}} a(t, y) \leq c_{E} \inf _{(t, y) \in[0, T] \times \mathbb{R}} a(t, y) .
$$

- the function a is seven-times continuously differentiable in the y-variable and

$$
\begin{aligned}
& M_{Y, 1}=\max _{1 \leq i \leq 7} \sup _{(t, y) \in[0, T] \times \mathbb{R}}\left|\partial_{y}^{i} a(t, y)\right|<\infty, \\
& M_{Y, 0}=\max \left(M_{Y, 1}, \sup _{(t, y) \in[0, T] \times \mathbb{R}} a(t, y)\right)<\infty .
\end{aligned}
$$

- the function $h: \mathbb{R} \mapsto \mathbb{R}$ is a.e. differentiable. In addition, $h$ and $h^{\prime}$ have at most an exponential growth: $|h(x)|+\left|h^{\prime}(x)\right| \leq c_{h} e^{c_{h}|x|}$ for any $x$, for a constant $c_{h}$.

Define

- the Gaussian process $\left(Y_{t}^{P}\right)_{0 \leq t \leq T}$ by

$$
Y_{t}^{P}=Y_{0}-\frac{1}{2} \int_{0}^{t} a^{2}\left(s, Y_{0}\right) d s+\int_{0}^{t} a\left(s, Y_{0}\right) d W_{s}
$$

Page: 5 job:GOBET_SULEIMAN_VolLocale_FinalVersion macro: svmult.cls date/time: 5-0ct-2010/9:10 
- $a(t):=a\left(t, Y_{0}\right), a^{(1)}(t):=\partial_{y}^{1} a\left(t, Y_{0}\right)$ and $a^{(2)}(t):=\partial_{y}^{2} a\left(t, Y_{0}\right)$;

- the expansion coefficients computed using the function a $(t,$.$) at Y_{0}$ :

$$
\begin{array}{llrl}
c_{1, T} & =\omega\left(a^{2}, a a^{(1)}\right)_{0}^{T}, & c_{2, T} & =\omega\left(a^{2},\left(a^{(1)}\right)^{2}\right)_{0}^{T}, \\
c_{3, T} & =\omega\left(a^{2}, a a^{(2)}\right)_{0}^{T}, & c_{4, T} & =\omega\left(a^{2}, a^{2},\left(a^{(1)}\right)^{2}\right)_{0}^{T}, \\
c_{5, T} & =\omega\left(a^{2}, a^{2}, a a^{(2)}\right)_{0}^{T}, & c_{6, T} & =\omega\left(a^{2}, a a^{(1)}, a a^{(1)}\right)_{0}^{T}, \\
c_{7, T} & =\omega\left(a^{2}, a^{2}, a a^{(1)}, a a^{(1)}\right)_{0}^{T}, & & c_{8, T}=\omega\left(a^{2}, a a^{(1)}, a^{2}, a a^{(1)}\right)_{0}^{T} .
\end{array}
$$

Then, the following expansion formulas hold.

a) Second order approximation. One has

$$
\begin{aligned}
\mathbb{E}\left[h\left(Y_{T}\right)\right]=\mathbb{E}\left[h\left(Y_{T}^{P}\right)\right]+c_{1, T}\left(\frac{1}{2} \operatorname{Greek}_{1}^{h}\left(Y_{T}^{P}\right)\right. & \left.-\frac{3}{2} \operatorname{Greek}_{2}^{h}\left(Y_{T}^{P}\right)+\operatorname{Greek}_{3}^{h}\left(Y_{T}^{P}\right)\right) \\
& +\operatorname{Error}_{2},
\end{aligned}
$$

where

$$
\mid \text { Error }_{2} \mid \leq C \sup _{v \in[0,1]}\left\|h^{(1)}\left(v Y_{T}+(1-v) Y_{T}^{P}\right)\right\|_{2}\left(\frac{M_{Y, 0}}{a_{i n f}}\right) M_{Y, 1} M_{Y, 0}^{2} T^{3 / 2}
$$

and the constant $C$ depends (in an increasing way) only on the upper bounds of the model parameters, on $c_{E}$ and on the maturity.

b) Third order approximation. One has

$$
\mathbb{E}\left[h\left(Y_{T}\right)\right]=\mathbb{E}\left[h\left(Y_{T}^{P}\right)\right]+\sum_{i=1}^{6} \eta_{i, T} \operatorname{Greek}_{i}^{h}\left(Y_{T}^{P}\right)+\text { Error }_{3},
$$

where

$$
\begin{aligned}
& \eta_{1, T}=\frac{c_{1, T}}{2}-\frac{c_{2, T}}{2}-\frac{c_{3, T}}{2}-\frac{c_{4, T}}{4}-\frac{c_{5, T}}{4}-\frac{c_{6, T}}{2}, \\
& \eta_{2, T}=-\frac{3 c_{1, T}}{2}+\frac{c_{2, T}}{2}+\frac{c_{3, T}}{2}+\frac{5 c_{4, T}}{4}+\frac{5 c_{5, T}}{4}+\frac{7 c_{6, T}}{2}+\frac{c_{7, T}}{2}+\frac{c_{8, T}}{4}, \\
& \eta_{3, T}=c_{1, T}-2 c_{4, T}-2 c_{5, T}-6 c_{6, T}-3 c_{7, T}-\frac{3 c_{8, T}}{2}, \\
& \eta_{4, T}=c_{4, T}+c_{5, T}+3 c_{6, T}+\frac{13 c_{7, T}}{2}+\frac{13 c_{8, T}}{4} \\
& \eta_{5, T}=-6 c_{7, T}-3 c_{8, T}, \\
& \eta_{6, T}=2 c_{7, T}+c_{8, T},
\end{aligned}
$$

and

$$
\mid \text { Error }_{3} \mid \leq C \sup _{v \in[0,1]}\left\|h^{(1)}\left(v Y_{T}+(1-v) Y_{T}^{P}\right)\right\|_{2}\left(\frac{M_{Y, 0}}{a_{i n f}}\right)^{2} M_{Y, 1} M_{Y, 0}^{3} T^{2} .
$$

As before, the constant $C$ depends (in an increasing way) only on the upper bounds of the model parameters, on $c_{E}$ and on the maturity. 
As explained in [BGM10b], the approximation order is related to the power $m$ in the error upper bounds $M_{Y, 1} M_{Y, 0}^{m}(\sqrt{T})^{m+1}$. The smaller the volatility $\left(M_{Y, 0} \rightarrow 0\right)$ or its variations $\left(M_{Y, 1} \rightarrow 0\right)$ or the maturity $(T \rightarrow 0)$, the more accurate the approximations. Since the proxy is Gaussian, the computation of $\mathbb{E}\left[h\left(Y_{T}^{P}\right)\right]$ and Greek $_{i}^{h}\left(Y_{T}^{P}\right)$ can be performed in closed forms for usual functions $h$ (such as call/put payoffs), or by using efficient numerical integration techniques in other cases.

An interesting property of these expansion formulas is that they are exact for $h(x)=e^{x}$ (indeed $\mathbb{E}\left[h\left(Y_{T}\right)\right]=\mathbb{E}\left[h\left(Y_{T}^{P}\right)\right]=\operatorname{Greek}_{i}^{h}\left(Y_{T}^{P}\right)=e^{Y_{0}}$, and the sum of expansion coefficients is equal to 0$)$. In particular, when further applied to the local volatility model (1.1), this implies that the call/put parity will be preserved within these approximations.

When the function $(t, x) \mapsto a(t, x)$ is piecewise constant w.r.t. the time variable, the coefficients $\left(c_{i, T}\right)_{1 \leq i \leq 8}$ can be quickly and simultaneously computed for different maturities $\bar{T}$, using recursion (see [BGM09, Proposition 4.1]). In other situations, numerical integration is likely needed.

\subsubsection{Application to expansion formulas for call price}

We go back to the local volatility model (1.1) and to the evaluation of call options. In view of (1.4), the call price at time 0 is equal to

$$
\operatorname{Call}(T, K)=\mathbb{E}\left(e^{-\int_{0}^{T} r_{s} d s}\left(S_{T}-K\right)_{+}\right)=\mathbb{E}\left(h\left(X_{T}\right)\right)
$$

where $h(x)=e^{-\int_{0}^{T} r_{s} d s}\left(C_{T} e^{x}-K\right)_{+}$. In order to apply previous expansion results, it remains to identify the function $a(\cdot)$ in the dynamics of $d X_{t}=$ $a\left(t, X_{t}\right) d W_{t}-\frac{1}{2} a^{2}\left(t, X_{t}\right) d t$. Comparing with (1.4), it follows that

$$
a(t, x)=\sigma\left(t, C_{t} \exp (x)\right) .
$$

Owing to the assumptions $(R)$ and $(E)$ on $\sigma$, one can apply Theorem 1 to $Y=X$ and to $h(x)=e^{-\int_{0}^{T} r_{s} d s}\left(C_{T} e^{x}-K\right)_{+}$, in order to obtain expansion formulas for call prices in local volatility models. The next step consists in transforming the Greeks in the $X$-variable in the (usual) Greeks in the $S$ variable, and in expressing the coefficients $c_{i, T}$ using the derivatives of $\sigma$. These computations are detailed in Appendix A. We obtain the following

Theorem 2 (Second and third order approximations for call options, based on the ATM local volatility).

Assume $(E)$ and $(R)$. Set $\sigma_{t}:=\sigma\left(t, C_{t} S_{0}\right), \sigma_{t}^{(1)}:=\partial_{S} \sigma\left(t, C_{t} S_{0}\right), \sigma_{t}^{(2)}:=$ $\partial_{S}^{2} \sigma\left(t, C_{t} S_{0}\right)$ and

$$
\begin{array}{ll}
\alpha_{1, T}=\omega\left(\sigma^{2}, S_{0} C \sigma \sigma^{(1)}\right)_{0}^{T}, & \alpha_{2, T}=\omega\left(\sigma^{2},\left(S_{0} C \sigma^{(1)}\right)^{2}\right)_{0}^{T}, \\
\alpha_{3, T}=\omega\left(\sigma^{2}, S_{0}^{2} C^{2} \sigma \sigma^{(2)}+S_{0} C \sigma \sigma^{(1)}\right)_{0}^{T}, & \alpha_{4, T}=\omega\left(\sigma^{2}, \sigma^{2},\left(S_{0} C \sigma^{(1)}\right)^{2}\right)_{0}^{T}, \\
\alpha_{5, T}=\omega\left(\sigma^{2}, \sigma^{2}, S_{0}^{2} C^{2} \sigma \sigma^{(2)}+S_{0} C \sigma \sigma^{(1)}\right)_{0}^{T}, & \alpha_{6, T}=\omega\left(\sigma^{2}, S_{0} C \sigma \sigma^{(1)}, S_{0} C \sigma \sigma^{(1)}\right)_{0}^{T}, \\
\alpha_{7, T}=\omega\left(\sigma^{2}, \sigma^{2}, S_{0} C \sigma \sigma^{(1)}, S_{0} C \sigma \sigma^{(1)}\right)_{0}^{T}, & \alpha_{8, T}=\omega\left(\sigma^{2}, S_{0} C \sigma \sigma^{(1)}, \sigma^{2}, S_{0} C \sigma \sigma^{(1)}\right)_{0}^{T} .
\end{array}
$$

Page: 7 job:GOBET_SULEIMAN_VolLocale_FinalVersion macro: svmult.cls date/time: 5-0ct-2010/9:10 
a) Second order approximation. One has

$$
\begin{aligned}
& \operatorname{Call}(T, K)=\operatorname{Call}^{\mathrm{BS}}\left(0, S_{0} ; T, K\right) \\
& \quad+\alpha_{1, T}\left(\frac{3}{2} S_{0}^{2} \partial_{S}^{2} \mathrm{Call}^{\mathrm{BS}}\left(0, S_{0} ; T, K\right)+S_{0}^{3} \partial_{S}^{3} \mathrm{Call}^{\mathrm{BS}}\left(0, S_{0} ; T, K\right)\right)+\text { Error }_{2}, \\
& \mid \text { Error }_{2} \mid \leq C S_{0} \exp \left(-\frac{\left[\log \left(S_{0} C_{T} / K\right)\right]^{2}}{8|\sigma|_{\infty}^{2} T}\right)\left(\frac{M_{0}}{\sigma_{\text {inf }}}\right) M_{1} M_{0}^{2} T^{3 / 2}
\end{aligned}
$$

where the Black-Scholes price and greeks are computed using the time dependent parameters $\left(\sigma_{t}, r_{t}, q_{t}\right)_{0 \leq t \leq T}$.

b) Third order approximation. One has

$$
\begin{aligned}
\operatorname{Call}(T, K)= & \text { Call }^{\mathrm{BS}}\left(0, S_{0} ; T, K\right) \\
& +\sum_{i=2}^{6} \pi_{i, T} S_{0}^{i} \partial_{S}^{i} \mathrm{Call}^{\mathrm{BS}}\left(0, S_{0} ; T, K\right)+\text { Error }_{3},
\end{aligned}
$$

where

$$
\begin{aligned}
\pi_{2, T} & =\frac{3}{2} \alpha_{1, T}+\frac{1}{2} \alpha_{2, T}+\frac{1}{2} \alpha_{3, T}+\frac{9}{4} \alpha_{4, T}+\frac{9}{4} \alpha_{5, T}+\frac{13}{2} \alpha_{6, T}+9 \alpha_{7, T}+\frac{9}{2} \alpha_{8, T}, \\
\pi_{3, T} & =\alpha_{1, T}+4 \alpha_{4, T}+4 \alpha_{5, T}+12 \alpha_{6, T}+66 \alpha_{7, T}+33 \alpha_{8, T}, \\
\pi_{4, T} & =\alpha_{4, T}+\alpha_{5, T}+3 \alpha_{6, T}+\frac{153}{2} \alpha_{7, T}+\frac{153}{4} \alpha_{8, T} \\
\pi_{5, T} & =24 \alpha_{7, T}+12 \alpha_{8, T} \\
\pi_{6, T} & =2 \alpha_{7, T}+\alpha_{8, T}, \\
\mid \text { Error }_{3} \mid & \leq C S_{0} \exp \left(-\frac{\left[\log \left(S_{0} C_{T} / K\right)\right]^{2}}{8|\sigma|_{\infty}^{2} T}\right)\left(\frac{M_{0}}{\sigma_{i n f}}\right)^{2} M_{1} M_{0}^{3} T^{2} .
\end{aligned}
$$

In the above expansions, the constant $C$ depends (in an increasing way) only on the upper bounds of the model parameters, on $c_{E}$ and on the maturity.

Note that the local volatility and its derivatives are computed along the ATM forward curve $\left(S_{0} C_{t}\right)_{0 \leq t \leq T}$.

\subsubsection{Other expansions based on the local volatility at strike}

In the previous approximation formulas, the ATM local volatility plays a central role. This is quite natural for arbitrary functions $h$, like in the general form of Theorem 1. But when dealing with call-put options, the local volatility at strike presumably plays a similarly important role. The aim of this paragraph is to derive similar expansion formulas, but using the volatility at strike. To achieve this goal, we follow the Dupire approach [Dup94], which writes a PDE satisfied by the call price function $(T, K) \mapsto \operatorname{Call}(T, K)=$ $\mathbb{E}\left(e^{-\int_{0}^{T} r_{s} d s}\left(S_{T}-K\right)_{+}\right)$. Indeed, we know that 


$$
\begin{aligned}
\frac{\partial \operatorname{Call}(T, K)}{\partial T} & =-q_{T} \operatorname{Call}(T, K)-\left(r_{T}-q_{T}\right) K \frac{\partial \operatorname{Call}(T, K)}{\partial K}+\frac{1}{2} \sigma^{2}(T, K) K^{2} \frac{\partial^{2} \operatorname{Call}(T, K)}{\partial K^{2}}, \\
\operatorname{Call}(0, K) & =\left(S_{0}-K\right)_{+} .
\end{aligned}
$$

In other words, instead of handling a PDE in the backward variables $(t, S)$ with a call payoff as a terminal condition, we now deal with a PDE in the forward variables $(T, K)$, with a put payoff as an initial condition. This latter has a probabilistic Feynman-Kac representation

$$
\operatorname{Call}(T, K)=e^{-\int_{0}^{T} q_{T-t} d t} \mathbb{E}\left[\left(S_{0}-K_{T}\right)_{+}\right] .
$$

using the following diffusion process $\left(K_{t}\right)_{0 \leq t \leq T}$ :

$$
\frac{d K_{t}}{K_{t}}=-\left(r_{T-t}-q_{T-t}\right) d t+\sigma\left(T-t, K_{t}\right) d W_{t}, \quad K_{0}=K .
$$

Define the process $\left(Y_{t}\right)_{0 \leq t \leq T}$ as follows:

$$
K_{t}=e^{-\int_{0}^{t}\left(r_{T-s}-q_{T-s}\right) d s} e^{Y_{t}}=\frac{C_{T-t}}{C_{T}} e^{Y_{t}} .
$$

Then, $Y$ has a dynamics of the form (1.7) with $a(t, y)=\sigma\left(T-t, \frac{C_{T-t}}{C_{T}} e^{y}\right)$. Thus, we are in a position to apply the general Theorem 1, to $Y$ and to the function $h(y)=e^{-\int_{0}^{T} q_{T-t} d t}\left(S_{0}-\frac{C_{0}}{C_{T}} e^{y}\right)_{+}$. Retransforming the Greeks w.r.t. the $Y$-variable into usual Greeks w.r.t. $K$, we obtain the new following expansion formulas (see Appendix B for the proof).

Theorem 3 (Second and third order approximations for call options, based on the local volatility at strike).

Assume $(E)$ and $(R)$. Set $\tilde{C}_{t}=\frac{C_{T-t}}{C_{T}}, \tilde{\sigma}_{t}:=\sigma\left(T-t, \tilde{C}_{t} K\right), \tilde{\sigma}_{t}^{(1)}:=\partial_{S} \sigma(T-$ $\left.t, \tilde{C}_{t} K\right), \tilde{\sigma}_{t}^{(2)}=\partial_{S^{2}}^{2} \sigma\left(T-t, \tilde{C}_{t} K\right)$ and

$$
\begin{array}{ll}
\tilde{\alpha}_{1, T}=\omega\left(\tilde{\sigma}^{2}, K \tilde{C} \tilde{\sigma} \tilde{\sigma}^{(1)}\right)_{0}^{T}, & \tilde{\alpha}_{2, T}=\omega\left(\tilde{\sigma}^{2},\left(K \tilde{C} \tilde{\sigma}^{(1)}\right)^{2}\right)_{0}^{T}, \\
\tilde{\alpha}_{3, T}=\omega\left(\tilde{\sigma}^{2}, K^{2} \tilde{C}^{2} \tilde{\sigma} \tilde{\sigma}^{(2)}+K \tilde{C} \tilde{\sigma} \tilde{\sigma}^{(1)}\right)_{0}^{T}, & \tilde{\alpha}_{4, T}=\omega\left(\tilde{\sigma}^{2}, \tilde{\sigma}^{2},\left(K \tilde{C} \tilde{\sigma}^{(1)}\right)^{2}\right)_{0}^{T}, \\
\tilde{\alpha}_{5, T}=\omega\left(\tilde{\sigma}^{2}, \tilde{\sigma}^{2}, K^{2} \tilde{C}^{2} \tilde{\sigma} \tilde{\sigma}^{(2)}+K \tilde{C} \tilde{\sigma} \tilde{\sigma}^{(1)}\right)_{0}^{T}, & \alpha_{6, T}=\omega\left(\tilde{\sigma}^{2}, K \tilde{C} \tilde{\sigma} \tilde{\sigma}^{(1)}, K \tilde{C} \tilde{\sigma} \tilde{\sigma}^{(1)}\right)_{0}^{T}, \\
\tilde{\alpha}_{7, T}=\omega\left(\tilde{\sigma}^{2}, \tilde{\sigma}^{2}, K \tilde{C} \tilde{\sigma} \tilde{\sigma}^{(1)}, K \tilde{C} \tilde{\sigma} \tilde{\sigma}^{(1)}\right)_{0}^{T}, & \tilde{\alpha}_{8, T}=\omega\left(\tilde{\sigma}^{2}, K \tilde{C} \tilde{\sigma} \tilde{\sigma}^{(1)}, \tilde{\sigma}^{2}, K \tilde{C} \tilde{\sigma} \tilde{\sigma}^{(1)}\right)_{0}^{T} .
\end{array}
$$

a) Second order approximation. One has

$$
\begin{aligned}
& \operatorname{Call}(T, K)=\operatorname{Call}^{\mathrm{BS}}\left(0, S_{0} ; T, K\right) \\
& +\tilde{\alpha}_{1, T}\left(\frac{3}{2} K^{2} \partial_{K}^{2} \mathrm{Call}^{\mathrm{BS}}\left(0, S_{0} ; T, K\right)+K^{3} \partial_{K}^{3} \mathrm{Call}^{\mathrm{BS}}\left(0, S_{0} ; T, K\right)\right)+\text { Error }_{2} \\
& \mid \text { Error }_{2} \mid \leq C K \exp \left(-\frac{\left[\log \left(S_{0} C_{T} / K\right)\right]^{2}}{8|\sigma|_{\infty}^{2} T}\right)\left(\frac{M_{0}}{\sigma_{\text {inf }}}\right) M_{1} M_{0}^{2} T^{3 / 2}
\end{aligned}
$$


where the Black-Scholes price and greeks are computed using the time dependent parameters $\left(\tilde{\sigma}_{t}, r_{t}, q_{t}\right)_{0 \leq t \leq T}$.

b) Third order approximation. One has

$$
\begin{aligned}
\operatorname{Call}(T, K)= & \mathrm{Call}^{\mathrm{BS}}\left(0, S_{0} ; T, K\right) \\
& +\sum_{i=2}^{6} \tilde{\pi}_{i, T} K^{i} \partial_{K}^{i} \mathrm{Call}^{\mathrm{BS}}\left(0, S_{0} ; T, K\right)+\text { Error }_{3},
\end{aligned}
$$

where

$$
\begin{aligned}
\tilde{\pi}_{2, T} & =\frac{3}{2} \tilde{\alpha}_{1, T}+\frac{1}{2} \tilde{\alpha}_{2, T}+\frac{1}{2} \tilde{\alpha}_{3, T}+\frac{9}{4} \tilde{\alpha}_{4, T}+\frac{9}{4} \tilde{\alpha}_{5, T}+\frac{13}{2} \tilde{\alpha}_{6, T}+9 \tilde{\alpha}_{7, T}+\frac{9}{2} \tilde{\alpha}_{8, T}, \\
\tilde{\pi}_{3, T} & =\tilde{\alpha}_{1, T}+4 \tilde{\alpha}_{4, T}+4 \tilde{\alpha}_{5, T}+12 \tilde{\alpha}_{6, T}+66 \tilde{\alpha}_{7, T}+33 \tilde{\alpha}_{8, T}, \\
\tilde{\pi}_{4, T} & =\tilde{\alpha}_{4, T}+\tilde{\alpha}_{5, T}+3 \tilde{\alpha}_{6, T}+\frac{153}{2} \tilde{\alpha}_{7, T}+\frac{153}{4} \tilde{\alpha}_{8, T}, \\
\tilde{\pi}_{5, T} & =24 \tilde{\alpha}_{7, T}+12 \tilde{\alpha}_{8, T}, \\
\tilde{\pi}_{6, T} & =2 \tilde{\alpha}_{7, T}+\tilde{\alpha}_{8, T}, \\
\mid \text { Error }_{3} \mid & \leq C K \exp \left(-\frac{\left[\log \left(S_{0} C_{T} / K\right)\right]^{2}}{8|\sigma|_{\infty}^{2} T}\right)\left(\frac{M_{0}}{\sigma_{i n f}}\right)^{2} M_{1} M_{0}^{3} T^{2} .
\end{aligned}
$$

In the above expansions, the constant $C$ depends (in an increasing way) only on the upper bounds of the model parameters, on $c_{E}$ and on the maturity.

\subsubsection{Expansion formulas for implied volatility}

Interestingly, the previous expansions of call price can be turned into expansions of implied volatility $\sigma^{I}\left(0, S_{0} ; T, K\right)$ defined by $\operatorname{Call}(T, K)=$ $\operatorname{Call}^{\mathrm{BS}}\left(0, S_{0} ; T, K ; \sigma^{I}\left(0, S_{0} ; T, K\right),\left(r_{t}\right)_{0 \leq t \leq T},\left(q_{t}\right)_{0 \leq t \leq T}\right)$. To achieve this, we use nice relations between Greeks (see below and Proposition 1), omitting to indicate all the parameters whenever unambiguous:

$$
\begin{aligned}
\text { Vega } & =\frac{\partial}{\partial \sigma} \operatorname{Call}^{\mathrm{BS}}(0, S ; T, K)=S e^{-q T} \mathcal{N}^{\prime}\left(d_{1}\right) \sqrt{T}=K e^{-r T} \mathcal{N}^{\prime}\left(d_{2}\right) \sqrt{T}, \\
S^{2} \Gamma_{\mathrm{S}} & =S^{2} \frac{\partial^{2}}{\partial S^{2}} \operatorname{Call}^{\mathrm{BS}}(0, S ; T, K)=\frac{S e^{-q T} \mathcal{N}^{\prime}\left(d_{1}\right)}{\sigma \sqrt{T}}=\frac{\text { Vega }}{\sigma T}, \\
S^{3} \text { Speed }_{\mathrm{S}} & =S^{3} \frac{\partial^{3}}{\partial S^{3}} \operatorname{Call}^{\mathrm{BS}}(0, S ; T, K)=-S^{2} \Gamma_{\mathrm{S}}\left(\frac{d_{1}}{\sigma \sqrt{T}}+1\right)=-\frac{\text { Vega }}{\sigma T}\left(\frac{d_{1}}{\sigma \sqrt{T}}+1\right), \\
K^{2} \Gamma_{\mathrm{K}} & =\frac{\partial^{2}}{\partial K^{2}} \operatorname{Call}^{\mathrm{BS}}(0, S ; T, K)=\frac{K e^{-r T} \mathcal{N}^{\prime}\left(d_{2}\right)}{\sigma \sqrt{T}}=\frac{\text { Vega }}{\sigma T}, \\
K^{3} \operatorname{Speed}_{\mathrm{K}} & =K^{3} \frac{\partial^{3}}{\partial K^{3}} \operatorname{Call}^{\mathrm{BS}}(0, S ; T, K)=-K^{2} \Gamma_{\mathrm{K}}\left(1-\frac{d_{2}}{\sigma \sqrt{T}}\right)=-\frac{\text { Vega }}{\sigma T}\left(1-\frac{d_{2}}{\sigma \sqrt{T}}\right) .
\end{aligned}
$$

Now, consider the second order expansion formula based on the ATM local volatility: it becomes 
$\operatorname{Call}(T, K)=\mathrm{Call}^{\mathrm{BS}}\left(0, S_{0} ; T, K\right)-\operatorname{Vega} \frac{\alpha_{1, T}}{\sqrt{T}\left(\int_{0}^{T} \sigma_{s}^{2} d s\right)^{3 / 2}} \log \left(\frac{S_{0} C_{T}}{K}\right)+$ Error $_{2}$.

This reads as an expansion of the implied volatility. The derivation is similar for the second order expansion formula based on the local volatility at strike. This proves the following

Theorem 4 (Second order approximations on implied volatilities). Assume (E) and $(R)$. Using the notations of Theorems 2 and 3, we have

$$
\begin{aligned}
& \sigma^{I}\left(0, S_{0} ; T, K\right)=\left(\frac{1}{T} \int_{0}^{T} \sigma_{s}^{2} d s\right)^{\frac{1}{2}}-\frac{\alpha_{1, T}}{T^{\frac{1}{2}}\left(\int_{0}^{T} \sigma_{s}^{2} d s\right)^{\frac{3}{2}}} \log \left(\frac{S_{0} C_{T}}{K}\right) \\
& +\operatorname{Error}_{2}^{I} \text {, } \\
& \sigma^{I}\left(0, S_{0} ; T, K\right)=\left(\frac{1}{T} \int_{0}^{T} \tilde{\sigma}_{s}^{2} d s\right)^{\frac{1}{2}}+\frac{\tilde{\alpha}_{1, T}}{T^{\frac{1}{2}}\left(\int_{0}^{T} \tilde{\sigma}_{s}^{2} d s\right)^{\frac{3}{2}}} \log \left(\frac{S_{0} C_{T}}{K}\right) \\
& +{\widetilde{\text { Error }_{2}}}^{I} \text {. }
\end{aligned}
$$

Note that in the first case (1.18), the local volatility is computed ATM, while in the second one (1.19), it is computed at strike.

In addition to these direct implied volatility approximations, one can upper bound the residual terms Error ${ }_{2}^{I}$ and $\widetilde{\text { Error }_{2}}$, simply applying the error estimates from Theorems 2 and 3 . We do not give the details of this derivation. As it can be expected, the error estimates depend on the ratio $\frac{\log \left(S_{0} C_{T} / K\right)}{|\sigma|_{\infty} \sqrt{T}}$, but actually, they are locally uniform w.r.t. this ratio. More precisely, for any $\xi>0$, there is a constant $C_{\xi}$ which depends (in an increasing way) on $\xi$, on the upper bounds of the model parameters, on $c_{E}$, on the maturity and on the ratio $M_{0} / \sigma_{\text {inf }}$ such that for any $S_{0}$ and $K$ satisfying $\left|\log \left(S_{0} C_{T} / K\right)\right| \leq \xi|\sigma|_{\infty} \sqrt{T}$ we have

$$
\left|\operatorname{Error}_{2}^{I}\right|+\left|{\widetilde{\operatorname{Error}_{2}}}^{I}\right| \leq C_{\xi} M_{1} M_{0}^{2} T .
$$

Thus, inaccuracies may occur for very small or very large strikes, a feature which is confirmed by the further numerical experiments. In view of the above upper bounds, the relative errors on implied volatility are locally of order $M_{1} M_{0} T$, justifying the label of second order approximations.

This paves the way for the derivation of a third order expansion of implied volatility, but unfortunately, we have not been able to simplify the computations in order to get a sufficiently nice expression. This will be further investigated.

\subsubsection{Applications to time-dependent CEV model}

To conclude this section, we specify the results when the volatility has the form

Page: 11 job:GOBET_SULEIMAN_VolLocale_FinalVersion macro: svmult.cls date/time: 5-0ct-2010/9:10 


$$
\sigma(t, S)=\nu_{t} S^{\beta_{t}-1}
$$

i.e. a CEV-type volatility with a time-dependent level $\left(\nu_{t}\right)_{0 \leq t \leq T}$ and a timedependent skew $\left(\beta_{t}\right)_{0 \leq t \leq T}$. In order to apply Theorems 2 and 3 , all what is needed is to give the expressions for the coefficients $\left(\alpha_{i, T}, \tilde{\alpha}_{i, T}\right)_{1 \leq i \leq 6}$. First, the proxy volatilities are given by $\sigma_{t}=\nu_{t}\left(C_{t} S_{0}\right)^{\beta_{t}-1}$ and $\tilde{\sigma}_{t}=\nu_{T-t}\left(\tilde{C}_{t} K\right)^{\beta_{T-t-1}}$, where $\tilde{C}_{t}=C_{T-t} / C_{T}$; then, we have

$$
\begin{array}{ll}
\alpha_{1, T}=\omega\left(\sigma^{2},(\beta-1) \sigma^{2}\right)_{0}^{T}, & \alpha_{2, T}=\alpha_{3, T}=\omega\left(\sigma^{2},(\beta-1)^{2} \sigma^{2}\right)_{0}^{T}, \\
\alpha_{4, T}=\alpha_{5, T}=\omega\left(\sigma^{2}, \sigma^{2},(\beta-1)^{2} \sigma^{2}\right)_{0}^{T}, & \alpha_{6, T}=\omega\left(\sigma^{2},(\beta-1) \sigma^{2},(\beta-1) \sigma^{2}\right)_{0}^{T}, \\
\alpha_{7, T}=\omega\left(\sigma^{2}, \sigma^{2},(\beta-1) \sigma^{2},(\beta-1) \sigma^{2}\right)_{0}^{T}, & \alpha_{8, T}=\omega\left(\sigma^{2},(\beta-1) \sigma^{2}, \sigma^{2},(\beta-1) \sigma^{2}\right)_{0}^{T} .
\end{array}
$$

The expressions are similar for $\left(\tilde{\alpha}_{i, T}\right)_{1 \leq i \leq 6}$, by replacing $\sigma_{t}$ by $\tilde{\sigma}_{t}$ and $\left(\beta_{t}-1\right)$ by $\left(\beta_{T-t}-1\right)$ in the above formulas. In the case of constant parameters $\nu_{t}=\nu$, $\beta_{t}=\beta$ and $\mu=r-q$, all the previous quantities can be expressed in closed forms (the values of the integral operator $\omega(.)_{0}^{T}$ are given by iterated integrals of exponential functions). We give them in the simple case $\mu=0$. By setting $\sigma=\nu S_{0}^{\beta-1}$ and $\tilde{\sigma}=\nu K^{\beta-1}$, we obtain

$$
\begin{array}{ll}
\alpha_{1, T}=(\beta-1) \sigma^{4} \frac{T^{2}}{2}, & \alpha_{2, T}=\alpha_{3, T}=(\beta-1)^{2} \sigma^{4} \frac{T^{2}}{2}, \\
\alpha_{4, T}=\alpha_{5, T}=\alpha_{6, T}=(\beta-1)^{2} \sigma^{6} \frac{T^{3}}{6}, & \alpha_{7, T}=\alpha_{8, T}=(\beta-1)^{2} \sigma^{8} \frac{T^{4}}{24} .
\end{array}
$$

Replacing $\sigma$ by $\tilde{\sigma}$ gives the values for $\left(\tilde{\alpha}_{i, T}\right)_{1 \leq i \leq 6}$.

\subsection{Numerical results}

In the numerical tests we report here, we take $r=q=0$ and we consider a CEV model (1.20) for the volatility, with constant parameters $\nu$ and $\beta$. For additional tests with time-dependent parameters, see [BGM10b]. We choose $S_{0}=1, \nu=25 \%$ and we allow $\beta$ to vary. Actually, we consider two values: $\beta=0.8$ which is not far from the log-normal case, and $\beta=0.2$ which is rather different. We test the accuracy of different approximations, for various maturities (3-6 months, 1-1.5-2-3-5-10 years) and various strikes.

\section{INSERT TABLE 1.1 ABOUT HERE}

The range of strikes depends on the maturity: the tested values are reported in Table 1.1. Essentially, the strikes are roughly equal to $S_{0} \exp (\xi \nu \sqrt{T})$ where $\xi$ is taken as various quantiles of the standard Gaussian law (we take the quantiles $1 \%-5 \%-10 \%-20 \%-30 \%-40 \%-50 \%-60 \%-70 \%-80 \%-90 \%-95 \%-99 \%)$ : this means that the first and last columns of strikes are associated to very ITM options or very OTM options.

For the sake of completeness, in Table 1.2 and 1.3 we report the implied volatilities related to the (exact) call price in CEV model with constant parameters (our computations are based on the work by Schroder [Sch89]).

Page: 12 job:GOBET_SULEIMAN_VolLocale_FinalVersion macro: svmult.cls date/time: 5-0ct-2010/9:10 


\section{INSERT TABLES 1.2 and 1.3 ABOUT HERE}

We aim at comparing the following different approximations.

1. ImpVol (AppPrice $(2, \mathrm{~S} 0)$ ): this is the implied volatility of the second order expansion based on the ATM local volatility (see (1.12) in Theorem 2).

2. AppImpVol $(2, \mathrm{~S} 0)$ : this is the second order implied volatility expansion based on the ATM local volatility (see (1.18) in Theorem 4).

3. ImpVol (AppPrice $(2, \mathrm{~K})$ ): this is the implied volatility of the second order expansion based on the local volatility at strike (see (1.16) in Theorem 3).

4. AppImpVol $(2, \mathrm{~K})$ : this is the second order implied volatility expansion based on the local volatility at strike (see (1.19) in Theorem 4).

5. ImpVol (AppPrice $(3, \mathrm{~S} 0)$ ): this is the implied volatility of the third order expansion based on the ATM local volatility (see (1.13) in Theorem 2).

6. ImpVol (AppPrice $(3, K))$ : this is the implied volatility of the third order expansion based on the local volatility at strike (see (1.17) in Theorem 3).

7. Av.ImpVol (AppPrice $(3,$.$) ): this is the average of ImpVol (AppPrice (3, \mathrm{SO})$ ) and ImpVol (AppPrice $(3, \mathrm{~K}))$. The interest in this approximation is explained later.

\section{INSERT TABLES 1.4 and 1.5 ABOUT HERE}

In Table 1.4 (resp. Table 1.5), we report the errors on implied volatility using the six first aforementioned approximations, for $\beta=0.8$ (resp. $\beta=0.2$ ). The errors are expressed in bps (basis points): an implied volatility of $25.01 \%$ instead of $25 \%$ yields $1 \mathrm{bp}$ error. For instance, on the first row of Table 1.4, the value -12.3 is associated to the approximation error of ImpVol (AppPrice $(2, \mathrm{~S} 0)$ ) for the first strike of maturity $T=3 M$ (i.e. $K=0.70)$; on the fourth row of Table 1.4, the value -0.9 refers to the approximation error of $\operatorname{AppImpVol}(2, \mathrm{~K})$ for the second strike of maturity $T=3 \mathrm{M}$ (i.e. $K=0.75$ ), and so one. Sometimes (especially for very small and very large strikes), the price approximation is out of the non-arbitrage interval for call options: in this case, one can not define a value for the implied volatility and we report ND in the tabular. For all these results, a medium (or large) error on implied volatility may yield a small (or reasonable) error on prices: this is especially true for ITM or OTM options, for which the Vega is small (see the discussion in [BGM10c]).

Influence of $\beta$ and $T$. Generally speaking, we observe that for $\beta=0.8$, the errors are smaller compared to $\beta=0.2$ : it is not surprising since the lognormal proxy suits better in the first case. This can also be explained by our error estimates, since $M_{1}$ is essentially proportional to $|\beta-1|$. Errors are increasing w.r.t. $T$, which is also coherent with our error estimates.

Influence of $K$. For usual values of strike (essentially in the Gaussian quantile range $[10 \%, 90 \%]$ ), errors are small (or very small, depending on the approximation that is used), usually smaller than $10 \mathrm{bps}$ for $\beta=0.8$ up to $10 \mathrm{Y}$

Page: 13 job:GOBET_SULEIMAN_VolLocale_FinalVersion macro: svmult.cls date/time: 5-0ct-2010/9:10 
maturity, and smaller than 20bps for $\beta=0.2$ up to maturity 5 Y. Error approximations on implied volatility are much larger for very ITM or very OTM options. For these situations, it may be a good idea to incorporate known asymptotic on the implied volatility (see for instance [Lee04]).

Influence of the type of approximation. Regarding the second order approximations, within this model it gives lower bounds on implied volatility (and on price). This systematic underestimation is a drawback of these approximations. Notice that it is usually much better to use the direct approximation on implied volatility (Theorem 4) compared to the implied volatility of the price approximation. However, these implied volatility expansions underestimate the true value as well.

As expected, third order approximations are more accurate than second order ones. The improvement is more significant for $\beta=0.2$. In Figures 1.1 and 1.2, we plot the errors on implied volatility for the maturity $T=1.5 Y$ (this choice is unimportant) for both values of $\beta$.

\section{INSERT FIGURES 1.1 AND 1.2 ABOUT HERE}

We first observe that ImpVol (AppPrice $(3, \mathrm{~S} 0)$ ) overestimates the true value for $K \gg S_{0}$ and yields an underestimation for $K \ll S_{0}$. This is the converse regarding ImpVol (AppPrice $(3, \mathrm{~K}))$. On Tables 1.4 and 1.5, we can check that this is generally satisfied for any maturity. Thus, an heuristic rule may be to consider the following confidence interval for the exact implied volatility:

$$
\sigma^{I}\left(0, S_{0} ; T, K\right) \in(\operatorname{ImpVol}(\operatorname{AppPrice}(3, \mathrm{~K})), \operatorname{ImpVol}(\operatorname{AppPrice}(3, \mathrm{~S} 0))) .
$$

If the width of this interval is too large, it somehow indicates an inaccuracy in our approximations.

Secondly, we observe that the errors using ImpVol (AppPrice $(3, \mathrm{~S} 0)$ ) and ImpVol (AppPrice $(3, \mathrm{~K})$ ) have roughly the same magnitude (but with opposite signs). Then, if we define the average

$$
\begin{aligned}
& \text { Av.ImpVol }(\operatorname{AppPrice}(3, .)) \\
& =\frac{1}{2}(\operatorname{ImpVol}(\operatorname{AppPrice}(3, \mathrm{~S} 0))+\operatorname{ImpVol}(\operatorname{AppPrice}(3, \mathrm{~K}))),
\end{aligned}
$$

we expect to obtain a much better implied volatility estimate. The errors for Av.ImpVol(AppPrice(3,.)) for $\beta=0.8$ and $\beta=0.2$ are reported in Tables 1.6 and 1.7. Observe that for maturities smaller than $5 \mathrm{Y}$, the accuracy is truly excellent (i.e. smaller than few bps) for a widened range of strikes.

\section{INSERT TABLES 1.6 and 1.7 ABOUT HERE}

We have compared our approximations with the known implied volatility approximation in the CEV model (with zero interest rates and zero dividend) (see [Hen08, formula (5.41) p.141]):

$$
\sigma^{I}\left(0, S_{0} ; T, K\right) \approx \nu \frac{(1-\beta) \ln \left(K / S_{0}\right)}{K^{1-\beta}-S_{0}^{1-\beta}}\left(1+\frac{(\beta-1)^{2} \nu^{2} T}{24}\left(\frac{S_{0}+K}{2}\right)^{2 \beta-2}\right) .
$$

Page: 14 job:GOBET_SULEIMAN_VolLocale_FinalVersion macro: svmult.cls date/time: 5-0ct-2010/9:10 
This latter approximation yields a slightly better numerical accuracy compared to ours (and it is quicker to evaluate). However, our approximations are also able to deal naturally with general time-dependent local volatility (with piecewise continuity in time), as a difference with [Hen08, Chapter 5] for instance, or with stochastic interest rates [BGM10a]. This may be a significant advantage compared to other approaches, while maintaining tight error estimates.

\section{A Proof of Theorem 2}

We apply Theorem 1 , by taking $h(x)=e^{-\int_{0}^{T} r_{s} d s}\left(C_{T} e^{x}-K\right)_{+}$and $a(t, x)=$ $\sigma\left(t, C_{t} \exp (x)\right)$. The required assumptions on $h$ and $a$ are satisfied owing to assumptions $(E)$ and $(R)$. By simple computations, we easily check that $M_{Y, 0}=M_{0}$ and $M_{Y, 1}=M_{1}$. The proxy of $X$ used in Theorem 1 now writes $X_{t}^{P}=\log \left(S_{0}\right)-\frac{1}{2} \int_{0}^{t} \sigma_{s}^{2} d s+\int_{0}^{t} \sigma_{s} d W_{s}$.

Main term and correction terms. From this, we deduce that the main term $\mathbb{E}\left(h\left(X_{T}^{P}\right)\right)$ in the expansion is equal to

$\mathbb{E}\left(e^{-\int_{0}^{T} r_{s} d s}\left(C_{T} e^{X_{T}^{P}}-K\right)_{+}\right)=\operatorname{Call}^{\mathrm{BS}}\left(0, S_{0} ; T, K ;\left(\sigma_{t}\right)_{0 \leq t \leq T},\left(r_{t}\right)_{0 \leq t \leq T},\left(q_{t}\right)_{0 \leq t \leq T}\right)$.

In the following, for the sake of brevity, we omit to indicate in the BlackScholes formula the dependence w.r.t. $\left(\sigma_{t}, r_{t}, q_{t}\right)_{0 \leq t \leq T}$. For computing the sensitivities $\operatorname{Greek}_{i}^{h}\left(X_{T}^{P}\right)=\left.\partial_{x}^{i} \mathbb{E}\left(h\left(X_{T}^{P}+x\right)\right)\right|_{x=0}$, we proceed similarly to the main term. First, we have $\mathbb{E}\left(h\left(X_{T}^{P}+x\right)\right)=\mathrm{Call}^{\mathrm{BS}}\left(0, S_{0} e^{x} ; T, K\right)$. By successive differentiations, we obtain (using matrix notation)

$$
\left(\begin{array}{l}
\operatorname{Greek}_{1}^{h}\left(X_{T}^{P}\right) \\
\operatorname{Greek}_{2}^{h}\left(X_{T}^{P}\right) \\
\operatorname{Greek}_{3}^{h}\left(X_{T}^{P}\right) \\
\operatorname{Greek}_{4}^{h}\left(X_{T}^{P}\right) \\
\operatorname{Greek}_{5}^{h}\left(X_{T}^{P}\right) \\
\operatorname{Greek}_{6}^{h}\left(X_{T}^{P}\right)
\end{array}\right)=\left(\begin{array}{cccccc}
1 & 0 & 0 & 0 & 0 & 0 \\
1 & 1 & 0 & 0 & 0 & 0 \\
1 & 3 & 1 & 0 & 0 & 0 \\
1 & 7 & 6 & 1 & 0 & 0 \\
1 & 15 & 25 & 10 & 1 & 0 \\
1 & 31 & 90 & 65 & 15 & 1
\end{array}\right)\left(\begin{array}{l}
S_{0} \partial_{S} \operatorname{Call}^{\mathrm{BS}}\left(0, S_{0} ; T, K\right) \\
S_{0}^{2} \partial_{S}^{2} \operatorname{Call}^{\mathrm{BS}}\left(0, S_{0} ; T, K\right) \\
S_{0}^{3} \partial_{S}^{3} \operatorname{Call}^{\mathrm{BS}}\left(0, S_{0} ; T, K\right) \\
S_{0}^{4} \partial_{S}^{4} \operatorname{Call}^{\mathrm{BS}}\left(0, S_{0} ; T, K\right) \\
S_{0}^{5} \partial_{S}^{5} \operatorname{Call}^{\mathrm{BS}}\left(0, S_{0} ; T, K\right) \\
S_{0}^{6} \partial_{S}^{6} \operatorname{Call}^{\mathrm{BS}}\left(0, S_{0} ; T, K\right)
\end{array}\right) .
$$

Regarding the summation of the correction terms, it implies that $\sum_{i=1}^{6} \eta_{i, T} \operatorname{Greek}_{i}^{h}\left(X_{T}^{P}\right)=$ $\sum_{i=1}^{6} \hat{\eta}_{i, T} S_{0}^{i} \partial_{S}^{i} \mathrm{Call}^{\mathrm{BS}}\left(0, S_{0} ; T, K\right)$ where

$$
\begin{aligned}
& \hat{\eta}_{1, T}=0, \\
& \hat{\eta}_{2, T}=\frac{3}{2} c_{1, T}+\frac{1}{2} c_{2, T}+\frac{1}{2} c_{3, T}+\frac{9}{4} c_{4, T}+\frac{9}{4} c_{5, T}+\frac{13}{2} c_{6, T}+9 c_{7, T}+\frac{9}{2} c_{8, T}, \\
& \hat{\eta}_{3, T}=c_{1, T}+4 c_{4, T}+4 c_{5, T}+12 c_{6, T}+66 c_{7, T}+33 c_{8, T}, \\
& \hat{\eta}_{4, T}=c_{4, T}+c_{5, T}+3 c_{6, T}+\frac{153}{2} c_{7, T}+\frac{153}{4} c_{8, T}, \\
& \hat{\eta}_{5, T}=24 c_{7, T}+12 c_{8, T}, \\
& \hat{\eta}_{6, T}=2 c_{7, T}+c_{8, T} .
\end{aligned}
$$


The expressions of the coefficients $\left(c_{i, T}\right)_{1 \leq i \leq 8}$ are given in Theorem 1 , but in order to specify them in the current case $a(t, x)=\sigma\left(t, C_{t} \exp (x)\right)$, we denote them by $\left(\alpha_{i, T}\right)_{1 \leq i \leq 8}$ instead of $\left(c_{i, T}\right)_{1 \leq i \leq 8}$. Easy computations show that these definitions coincide with those given in Theorem 2. Then, the second order expansion formula is obtained by keeping only the first coefficient $\alpha_{1, T}$, while all the coefficients are taken for the third order expansion formula.

Error estimates. We have already observed that $M_{Y, 0}=M_{0}$ and $M_{Y, 1}=$ $M_{1}$. It remains to estimate the factor $\left\|h^{(1)}\left(v X_{T}+(1-v) X_{T}^{P}\right)\right\|_{2}$ arising in the error bounds of Theorem 1. For $v \in[0,1]$, define $\sigma_{t}^{v}:=v \sigma\left(t, X_{t}\right)+(1-v) \sigma_{t} \in$ $\left[\sigma_{i n f},|\sigma|_{\infty}\right]$ and $\sigma_{t}^{2, v}:=v \sigma^{2}\left(t, X_{t}\right)+(1-v) \sigma_{t}^{2} \in\left[\sigma_{i n f}^{2},|\sigma|_{\infty}^{2}\right]:$ clearly we have

$$
d\left(v X_{t}+(1-v) X_{t}^{P}\right)=\sigma_{t}^{v} d W_{t}-\frac{1}{2} \sigma_{t}^{2, v} d t .
$$

We denote by $\mathbb{P}^{v}$ the probability measure under which $W_{t}^{v}=W_{t}-2 \int_{0}^{t} \sigma_{s}^{v} d s$ is a Brownian motion. Then, putting $d_{0}=\log \left(S_{0} C_{T} / K\right)$ and using $h^{\prime}(x)=$ $e^{-\int_{0}^{T} q_{s} d s} e^{x} \mathbf{1}_{x-\log \left(S_{0}\right)>-d_{0}}$, we obtain

$$
\begin{aligned}
& \mathbb{E}\left(\left[h^{\prime}\left(v X_{T}+(1-v) X_{T}^{P}\right)\right]^{2}\right) \\
& =S_{0}^{2} e^{-2 \int_{0}^{T} q_{s} d s} \mathbb{E}\left(e^{2 \int_{0}^{T} \sigma_{s}^{v} d W_{s}-\int_{0}^{T} \sigma_{s}^{2, v} d s} \mathbf{1}_{\int_{0}^{T} \sigma_{s}^{v} d W_{s}-\frac{1}{2} \int_{0}^{T} \sigma_{s}^{2, v} d s>-d_{0}}\right) \\
& =S_{0}^{2} e^{-2 \int_{0}^{T} q_{s} d s} \mathbb{E}^{v}\left(e^{2 \int_{0}^{T}\left[\sigma_{s}^{v}\right]^{2} d s-\int_{0}^{T} \sigma_{s}^{2, v} d s} \mathbf{1}_{\int_{0}^{T} \sigma_{s}^{v} d W_{s}^{v}+2 \int_{0}^{T}\left[\sigma_{s}^{v}\right]^{2} d s-\frac{1}{2} \int_{0}^{T} \sigma_{s}^{2, v} d s>-d_{0}}\right) \\
& \leq S_{0}^{2} e^{-2 \int_{0}^{T} q_{s} d s+2|\sigma|_{\infty}^{2} T \mathbb{P}^{v}}\left(\int_{0}^{T} \sigma_{s}^{v} d W_{s}^{v}+2|\sigma|_{\infty}^{2} T>-d_{0}\right)
\end{aligned}
$$

If $-d_{0}>2|\sigma|_{\infty}^{2} T$, one can apply the Bernstein exponential inequality to show that the above probability is bounded by $\exp \left(-\frac{\left(-d_{0}-2|\sigma|_{\infty}^{2} T\right)^{2}}{2|\sigma|_{\infty}^{2} T}\right)$. Using the inequality $(a-b)^{2} \geq \frac{1}{2} a^{2}-b^{2}$, it follows that

$$
\sup _{v \in[0,1]} \mathbb{E}\left(\left[h^{\prime}\left(v X_{T}+(1-v) X_{T}^{P}\right)\right]^{2}\right) \leq C S_{0}^{2} \exp \left(-\frac{d_{0}^{2}}{4|\sigma|_{\infty}^{2} T}\right)
$$

where the constant $C$ depends in an increasing way on the bounds on the coefficients and on the maturity. Note that the inequality (1.24) is also valid if $0 \leq-d_{0} \leq 2|\sigma|_{\infty}^{2} T$ : indeed, from (1.23), we write $\mathbb{E}\left(\left[h^{\prime}\left(v X_{T}+(1-v) X_{T}^{P}\right)\right]^{2}\right) \leq$ $S_{0}^{2} e^{-2 \int_{0}^{T} q_{s} d s+2|\sigma|_{\infty}^{2} T} \leq S_{0}^{2} e^{-2 \int_{0}^{T} q_{s} d s+2|\sigma|_{\infty}^{2} T} \exp \left(\frac{\left(2|\sigma|_{\infty}^{2} T\right)^{2}}{4|\sigma|_{\infty}^{2} T}\right) \exp \left(-\frac{d_{0}^{2}}{4|\sigma|_{\infty}^{2} T}\right) \leq$ $C S_{0}^{2} \exp \left(-\frac{d_{0}^{2}}{4|\sigma|_{\infty}^{2} T}\right)$. To sum up we obtain

$$
\sup _{v \in[0,1]} \|\left[h^{\prime}\left(v X_{T}+(1-v) X_{T}^{P}\right) \|_{2} \leq C S_{0} \exp \left(-\frac{\left[\log \left(S_{0} C_{T} / K\right)\right]^{2}}{8|\sigma|_{\infty}^{2} T}\right)\right.
$$

for any $d_{0} \leq 0$, or equivalently for any $K \geq S_{0} C_{T}$. Thus, the announced estimates on Error 2 and Error 3 are valid for any Out of The Money calls. Using a similar analysis, the same estimates hold for Out of The Money puts ( $K \leq$ 
$\left.S_{0} C_{T}\right)$. But, since the call/put parity is preserved within these expansions, error estimates are equal for call/put with the same characteristics. Thus, estimates for Out of The Money puts transfer to In The Money calls. This completes the proof.

A careful inspection of the current proof and that of Theorem 1 reveals that the factor 8 in the exponential (1.25) can be improved and actually, it can be taken strictly larger than 2: this gives presumably better error estimates for $K \ll S_{0}$ or $K \gg S_{0}$.

\section{B Proof of Theorem 3}

The derivation of the expansion is obtained following the same lines as those for Theorem 2. We detail only the main arguments. The proxy for the process $\left(Y_{t}\right)_{0 \leq t \leq T}$ is defined by $Y_{t}^{P}=\log (K)+\int_{0}^{t} \tilde{\sigma}_{s} d W_{s}-\frac{1}{2} \int_{0}^{t} \tilde{\sigma}_{s}^{2} d s$. We interpret $e^{Y_{T}^{P}} / K$ as the Radon-Nikodym derivative of a new measure $\tilde{\mathbb{P}}$ w.r.t. $\mathbb{P}$ on $\mathcal{F}_{T}$, under which $\tilde{W}_{t}=W_{t}-\int_{0}^{t} \tilde{\sigma}_{s} d s$ is a standard BM; then we obtain

$$
\begin{aligned}
\mathbb{E}\left(e^{-\int_{0}^{T} q_{T-t} d t}\right. & \left.\left(S_{0}-\frac{C_{0}}{C_{T}} e^{Y_{T}^{P}}\right)_{+}\right)=\mathbb{E}\left(e^{-\int_{0}^{T} r_{s} d s} \frac{e^{Y_{T}^{P}}}{K}\left(S_{0} C_{T} \frac{K}{e^{Y_{T}^{P}}}-K\right)_{+}\right) \\
& =\widetilde{\mathbb{E}}\left(e^{-\int_{0}^{T} r_{s} d s}\left(S_{0} e^{\int_{0}^{T}\left(r_{s}-q_{s}\right) d s} e^{-\int_{0}^{T} \tilde{\sigma}_{s} d \tilde{W}_{s}-\frac{1}{2} \int_{0}^{T} \tilde{\sigma}_{s}^{2} d s}-K\right)_{+}\right) \\
& =\operatorname{Call}^{\mathrm{BS}}\left(0, S_{0} ; T, K ;\left(\tilde{\sigma}_{t}\right)_{0 \leq t \leq T},\left(r_{t}\right)_{0 \leq t \leq T},\left(q_{t}\right)_{0 \leq t \leq T}\right) .
\end{aligned}
$$

This gives the main term in the expansion. Regarding the computation of the sensitivities $\operatorname{Greek}_{i}^{h}\left(Y_{T}^{P}\right)$, observe that $\mathbb{E}\left(h\left(Y_{T}^{P}+x\right)\right)=\operatorname{Call}^{\mathrm{BS}}\left(0, S_{0} ; T, K e^{x}\right)$, omitting the last parameters $\left(\tilde{\sigma}_{t}, r_{t}, q_{t}\right)_{0 \leq t \leq T}$. Thus, we easily relate the sensitivities $\operatorname{Greek}_{i}^{h}\left(Y_{T}^{P}\right)$ to the Greeks of $\mathrm{Call}^{\mathrm{BS}}\left(0, S_{0} ; T, K\right)$ w.r.t. $K$ (instead of $S_{0}$ in the Theorem 2). The relation is affine and is similar to (1.22). The other steps of the proof are analogous to that of Theorem 2, replacing $S_{0}$ and $\sigma$ by $K$ and $\tilde{\sigma}$ in most places.

\section{Computations of derivatives of the Black-Scholes price function w.r.t. $S$ and $K$}

In the following proposition, we make explicit the formulas for the six first derivatives of $\mathrm{Call}^{\mathrm{BS}}(0, S ; T, K ; \sigma, r, q)$ (in short $\mathrm{Call}^{\mathrm{BS}}(0, S ; T, K)$ ) w.r.t. $S$ and $K$, leaving the proofs to the reader. These formulas are necessary to implement the expansions of Theorems 2 and 3.

Proposition 1 (Black-Scholes Greeks). Using the notation from Definition 3, the sensitivities w.r.t. $S$ are given by 


$$
\begin{aligned}
\Delta_{\mathrm{S}}(t, S ; T, K) & =\frac{\partial}{\partial S} \operatorname{Call}^{\mathrm{BS}}(t, S ; T, K)=e^{-q(T-t)} \mathcal{N}\left(d_{1}\right), \\
\Gamma_{\mathrm{S}}(t, S ; T, K) & =\frac{\partial^{2}}{\partial S^{2}} \operatorname{Call}^{\mathrm{BS}}(t, S ; T, K)=e^{-q(T-t)} \frac{\mathcal{N}^{\prime}\left(d_{1}\right)}{S \sigma \sqrt{T-t}}, \\
\operatorname{Speed}_{\mathrm{S}}(t, S ; T, K) & =\frac{\partial^{3}}{\partial S^{3}} \operatorname{Call}^{\mathrm{BS}}(t, S ; T, K)=-\frac{\Gamma_{\mathrm{S}}}{S}\left(\frac{d_{1}}{\sigma \sqrt{T-t}}+1\right), \\
\frac{\partial^{4}}{\partial S^{4}} \mathrm{Call}^{\mathrm{BS}}(t, S ; T, K) & =\frac{\Gamma_{\mathrm{S}}}{S^{2}}\left(2+\frac{3 d_{1}}{\sigma \sqrt{T-t}}+\frac{d_{1}^{2}-1}{\sigma^{2}(T-t)}\right), \\
\frac{\partial^{5}}{\partial S^{5}} \mathrm{Call}^{\mathrm{BS}}(t, S ; T, K) & =\frac{\Gamma_{\mathrm{S}}}{S^{3}}\left(-6-\frac{11 d_{1}}{\sigma \sqrt{T-t}}+\frac{6\left(1-d_{1}^{2}\right)}{\sigma^{2}(T-t)}+\frac{3 d_{1}-d_{1}^{3}}{\sigma^{3}(T-t)^{\frac{3}{2}}}\right), \\
\frac{\partial^{6}}{\partial S^{6}} \mathrm{Call}^{\mathrm{BS}}(t, S ; T, K) & =\frac{\Gamma_{\mathrm{S}}}{S^{4}}\left(24+\frac{50 d_{1}}{\sigma \sqrt{T-t}}+\frac{35\left(d_{1}^{2}-1\right)}{\sigma^{2}(T-t)}+\frac{10 d_{1}\left(d_{1}^{2}-3\right)}{\sigma^{3}(T-t)^{\frac{3}{2}}}+\frac{3\left(1-2 d_{1}^{2}\right)+d_{1}^{4}}{\sigma^{4}(T-t)^{2}}\right) .
\end{aligned}
$$

The sensitivities w.r.t. $K$ are given by

$$
\begin{aligned}
\Delta_{\mathrm{K}}(t, S ; T, K) & =\frac{\partial}{\partial K} \mathrm{Call}^{\mathrm{BS}}(t, S ; T, K)=-e^{-r(T-t)} \mathcal{N}\left(d_{2}\right), \\
\Gamma_{\mathrm{K}}(t, S ; T, K) & =\frac{\partial^{2}}{\partial K^{2}} \mathrm{Call}^{\mathrm{BS}}(t, S ; T, K)=e^{-r(T-t)} \frac{\mathcal{N}^{\prime}\left(d_{2}\right)}{K \sigma \sqrt{T-t}}, \\
\operatorname{Speed}_{\mathrm{K}}(t, S ; T, K) & =\frac{\partial^{3}}{\partial K^{3}} \operatorname{Call}^{\mathrm{BS}}(t, S ; T, K)=-\frac{\Gamma_{\mathrm{K}}}{K}\left(1-\frac{d_{2}}{\sigma \sqrt{T-t}}\right), \\
\frac{\partial^{4}}{\partial K^{4}} \mathrm{Call}^{\mathrm{BS}}(t, S ; T, K) & =\frac{\Gamma_{\mathrm{K}}}{K^{2}}\left(2-\frac{3 d_{2}}{\sigma \sqrt{T-t}}+\frac{d_{2}^{2}-1}{\sigma^{2}(T-t)}\right), \\
\frac{\partial^{5}}{\partial K^{5}} \mathrm{Call}^{\mathrm{BS}}(t, S ; T, K) & =\frac{\Gamma_{\mathrm{K}}}{K^{3}}\left(-6+\frac{11 d_{2}}{\sigma \sqrt{T-t}}+\frac{6\left(1-d_{2}^{2}\right)}{\sigma^{2}(T-t)}+\frac{d_{2}^{3}-3 d_{2}}{\sigma^{3}(T-t)^{\frac{3}{2}}}\right), \\
\frac{\partial^{6}}{\partial K^{6}} \mathrm{Call}^{\mathrm{BS}}(t, S ; T, K) & =\frac{\Gamma_{\mathrm{K}}}{K^{4}}\left(24-\frac{50 d_{2}}{\sigma \sqrt{T-t}}+\frac{35\left(d_{2}^{2}-1\right)}{\sigma^{2}(T-t)}-\frac{10 d_{2}\left(d_{2}^{2}-3\right)}{\sigma^{3}(T-t)^{\frac{3}{2}}}+\frac{3\left(1-2 d_{2}^{2}\right)+d_{2}^{4}}{\sigma^{4}(T-t)^{2}}\right) .
\end{aligned}
$$

\section{References}

[BGM09] E. Benhamou, E. Gobet, and M. Miri. Smart expansion and fast calibration for jump diffusion. Finance and Stochastics, 13(4):563-589, 2009.

[BGM10a] E. Benhamou, E. Gobet, and M. Miri. Analytical formulas for local volatility model with stochastic rates. To appear in Quantitative Finance, 2010.

[BGM10b] E. Benhamou, E. Gobet, and M. Miri. Expansion formulas for European options in a local volatility model. International Journal of Theoretical and Applied Finance, 13(4):603-634, 2010.

[BGM10c] E. Benhamou, E. Gobet, and M. Miri. Time dependent Heston model. SIAM Journal on Financial Mathematics, 1:289-325, 2010.

[Dup94] B. Dupire. Pricing with a smile. Risk, 7(1):18-20, 1994.

[EG10] P. Etore and E. Gobet. Stochastic expansion for the pricing of call options with discrete dividends. Submitted, 2010. 
[Hen08] P. Henry-Labordère. Analysis, Geometry, and Modeling in Finance: Advanced Methods in Option Pricing. Chapman and Hall, 2008.

[HW99] P. Hagan and D. Woodward. Equivalent Black volatilities. Applied Mathematical Finance, 6:147-157, 1999.

[Lee04] R.W. Lee. The moment formula for implied volatility at extreme strikes. Mathematical Finance, 14(3):469-480, 2004.

[MR98] M. Musiela and M. Rutkowski. Martingale methods in financial modelling. Springer Verlag, 1998.

[Pit05] V.V. Piterbarg. Stochastic volatility model with time-dependent skew. Applied Mathematical Finance, 12(2):147-185, 2005.

[Sch89] M. Schroder. Computing the constant elasticity of variance option pricing formula. Journal of Finance, 44:211-219, 1989.

\begin{tabular}{|c||c|c|c|c|c|c|c|c|c|c|c|c|c|}
\hline \multicolumn{1}{|c||}{$T$} & \multicolumn{10}{c|}{$K$} \\
\hline $3 \mathrm{M}$ & 0.70 & 0.75 & 0.80 & 0.85 & 0.90 & 0.95 & 1.00 & 1.05 & 1.10 & 1.20 & 1.25 & 1.30 & 1.35 \\
\hline $6 \mathrm{M}$ & 0.65 & 0.75 & 0.80 & 0.85 & 0.90 & 0.95 & 1.00 & 1.05 & 1.10 & 1.15 & 1.25 & 1.35 & 1.50 \\
\hline $1 \mathrm{Y}$ & 0.55 & 0.65 & 0.75 & 0.80 & 0.90 & 0.95 & 1.00 & 1.05 & 1.15 & 1.25 & 1.40 & 1.50 & 1.80 \\
\hline $1.5 \mathrm{Y}$ & 0.50 & 0.60 & 0.70 & 0.75 & 0.85 & 0.95 & 1.00 & 1.10 & 1.15 & 1.30 & 1.50 & 1.65 & 2.00 \\
\hline $2 \mathrm{Y}$ & 0.45 & 0.55 & 0.65 & 0.75 & 0.85 & 0.90 & 1.00 & 1.10 & 1.20 & 1.35 & 1.55 & 1.80 & 2.30 \\
\hline $3 \mathrm{Y}$ & 0.35 & 0.50 & 0.55 & 0.70 & 0.80 & 0.90 & 1.00 & 1.10 & 1.25 & 1.45 & 1.75 & 2.05 & 2.70 \\
\hline $5 \mathrm{Y}$ & 0.25 & 0.40 & 0.50 & 0.60 & 0.75 & 0.85 & 1.00 & 1.15 & 1.35 & 1.60 & 2.05 & 2.50 & 3.60 \\
\hline $10 \mathrm{Y}$ & 0.15 & 0.25 & 0.35 & 0.50 & 0.65 & 0.80 & 1.00 & 1.20 & 1.50 & 1.95 & 2.75 & 3.65 & 6.30 \\
\hline
\end{tabular}

Table 1.1. Set of maturities and strikes used for the numerical tests.

\begin{tabular}{|c||c|c|c|c|c|c|c|c|c|c|c|c|c|}
\hline $3 \mathrm{M}$ & 25.908 & 25.728 & 25.563 & 25.409 & 25.265 & 25.129 & 25.001 & 24.879 & 24.763 & 24.548 & 24.447 & 24.350 & 24.258 \\
\hline $6 \mathrm{M}$ & 26.096 & 25.728 & 25.564 & 25.410 & 25.266 & 25.130 & 25.001 & 24.880 & 24.764 & 24.654 & 24.448 & 24.258 & 24.001 \\
\hline 1Y & 26.530 & 26.096 & 25.729 & 25.565 & 25.267 & 25.131 & 25.003 & 24.881 & 24.655 & 24.449 & 24.171 & 24.002 & 23.562 \\
\hline $1.5 \mathrm{Y}$ & 26.780 & 26.304 & 25.907 & 25.731 & 25.413 & 25.133 & 25.004 & 24.766 & 24.656 & 24.353 & 24.003 & 23.772 & 23.311 \\
\hline $2 \mathrm{Y}$ & 27.058 & 26.531 & 26.099 & 25.732 & 25.414 & 25.270 & 25.005 & 24.768 & 24.552 & 24.262 & 23.925 & 23.564 & 22.980 \\
\hline $3 \mathrm{Y}$ & 27.729 & 26.783 & 26.534 & 25.911 & 25.570 & 25.272 & 25.008 & 24.770 & 24.453 & 24.089 & 23.633 & 23.254 & 22.605 \\
\hline $5 \mathrm{Y}$ & 28.646 & 27.377 & 26.788 & 26.313 & 25.739 & 25.421 & 25.012 & 24.664 & 24.268 & 23.854 & 23.258 & 22.788 & 21.943 \\
\hline $10 \mathrm{Y}$ & 30.079 & 28.658 & 27.746 & 26.800 & 26.118 & 25.586 & 25.022 & 24.568 & 24.020 & 23.386 & 22.573 & 21.918 & 20.694 \\
\hline
\end{tabular}

Table 1.2. CEV model $(\beta=0.8)$ : implied volatilities in $\%$. 


\begin{tabular}{|c||c|c|c|c|c|c|c|c|c|c|c|c|c|}
\hline $3 \mathrm{M}$ & 28.755 & 28.003 & 27.312 & 26.673 & 26.080 & 25.528 & 25.010 & 24.535 & 24.074 & 23.232 & 22.845 & 22.477 & 22.128 \\
\hline $6 \mathrm{M}$ & 29.590 & 28.017 & 27.325 & 26.686 & 26.092 & 25.539 & 25.021 & 24.535 & 24.078 & 23.646 & 22.851 & 22.133 & 21.177 \\
\hline $1 \mathrm{Y}$ & 31.537 & 29.624 & 28.046 & 27.352 & 26.116 & 25.561 & 25.042 & 24.555 & 23.664 & 22.867 & 21.814 & 21.189 & 19.602 \\
\hline $1.5 \mathrm{Y}$ & 32.706 & 30.568 & 28.831 & 28.075 & 26.736 & 25.583 & 25.062 & 24.115 & 23.681 & 22.513 & 21.202 & 20.359 & 18.733 \\
\hline $2 \mathrm{Y}$ & 34.034 & 31.618 & 29.692 & 28.103 & 26.761 & 26.163 & 25.083 & 24.133 & 23.288 & 22.177 & 20.921 & 19.621 & 17.619 \\
\hline $3 \mathrm{Y}$ & 37.339 & 32.840 & 31.698 & 28.924 & 27.459 & 26.209 & 25.124 & 24.170 & 22.930 & 21.547 & 19.882 & 18.555 & 16.406 \\
\hline $5 \mathrm{Y}$ & 42.069 & 35.797 & 33.000 & 30.816 & 28.271 & 26.908 & 25.205 & 23.802 & 22.262 & 20.709 & 18.589 & 17.011 & 14.382 \\
\hline $10 \mathrm{Y}$ & 47.850 & 41.604 & 37.460 & 33.144 & 30.082 & 27.758 & 25.378 & 23.535 & 21.407 & 19.089 & 16.346 & 14.325 & 10.993 \\
\hline
\end{tabular}

Table 1.3. CEV model $(\beta=0.2)$ : implied volatilities in $\%$.

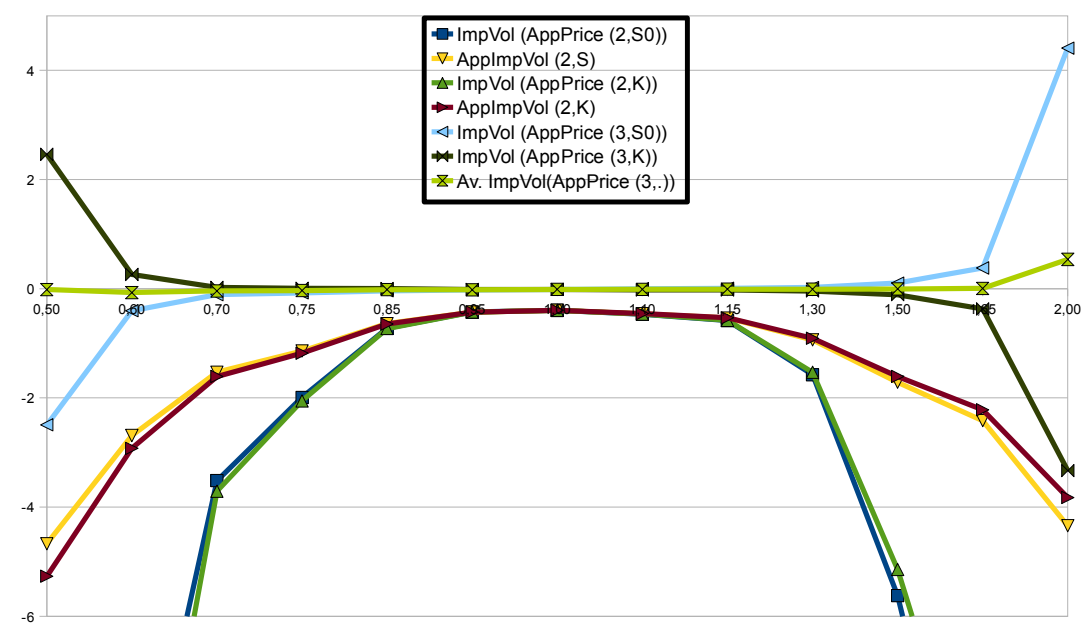

Fig. 1.1. CEV model $(\beta=0.8)$ : errors in bps on the implied volatility using the 7 approximations $\operatorname{ImpVol}(\operatorname{App} \operatorname{Price}(2, \mathrm{SO})), \operatorname{AppImpVol}(2, \mathrm{~S} 0)$, ImpVol (AppPrice $(2, \mathrm{~K})), \quad \operatorname{AppImpVol}(2, \mathrm{~K}), \quad \operatorname{ImpVol}(\operatorname{AppPrice}(3, \mathrm{~S} 0))$, ImpVol (AppPrice $(3, K))$ and Av. ImpVol $(\operatorname{AppPrice}(3,)$. 


\begin{tabular}{|c|c|c|c|c|c|c|c|c|c|c|c|c|c|}
\hline \multirow[t]{6}{*}{$3 \mathrm{M}$} & -12.3 & 5.8 & -2.4 & -0.9 & -0.3 & $|-0.1|$ & -0.1 & $|-0.1|$ & $\mid-0.2$ & $\mid-1.3$ & -2.6 & -4.9 & -8.5 \\
\hline & -1.7 & -0.9 & -0.5 & -0.3 & -0.2 & $|-0.1|$ & -0.1 & $-0.1 \mid$ & -0.1 & -0.3 & -0.5 & -0.6 & -08 \\
\hline & -17.1 & -6.8 & -2.6 & -0.9 & -0.3 & -0.1 & -0.1 & $|-0.1|$ & -0.2 & -1.2 & -2.4 & -4.2 & -6.8 \\
\hline & -1.7 & -0.9 & -0.5 & -0.3 & -0.2 & $-0.1 \mid$ & $-0.1 \mid$ & $-0.1 \mid$ & -0.1 & -0.3 & -0.5 & -0.6 & -0.8 \\
\hline & -1.4 & -0.4 & -0.1 & 0.0 & 0.0 & 0.0 & 0.0 & 0.0 & 0.0 & 0.0 & 0.1 & 0.1 & 0.4 \\
\hline & 0.6 & 0.1 & 0.0 & 0.0 & 0.0 & 0.0 & 0.0 & 0.0 & 0.0 & 0.0 & 0.0 & -0.1 & -0.4 \\
\hline \multirow[t]{6}{*}{$6 \mathrm{M}$} & -13.3 & -3.4 & -1.5 & -0.6 & 0.3 & \begin{tabular}{c|}
0.2 \\
\end{tabular} & 0.1 & $\mid-0.2$ & \begin{tabular}{|l|}
-0.2 \\
\end{tabular} & \begin{tabular}{|l|}
-0.4 \\
\end{tabular} & -1.6 & -4.4 & -14.8 \\
\hline & -1.9 & -0.9 & -0.6 & -0.4 & -0.2 & $-0.2 \mid$ & $-0.1 \mid$ & -0.2 & -0.2 & -0.3 & -0.5 & -0.9 & -1.5 \\
\hline & -17.7 & -3.7 & -1.6 & -0.7 & $|-0.3|$ & $-0.2 \mid$ & $|-0.1|$ & -0.2 & -0.2 & -0.4 & -1.5 & -4.0 & -11.2 \\
\hline & -2.1 & -0.9 & -0.6 & -0.4 & -0.2 & -0.2 & -0.1 & -0.2 & -0.2 & -0.3 & -0.5 & -0.8 & -1.4 \\
\hline & -1.1 & $-0.1]$ & 0.0 & 0.0 & 0.0 & 0.0 & 0.0 & 0.0 & 0.0 & 0.0 & 0.0 & 0.1 & 0.8 \\
\hline & 0.7 & 0.0 & 0.0 & 0.0 & 0.0 & 0.0 & 0.0 & 0.0 & 0.0 & 0.0 & 0.0 & -0.1 & -0.7 \\
\hline \multirow[t]{6}{*}{$1 \mathrm{Y}$} & -23.5 & -8.0 & -2.3 & -1.2 & -0.4 & -0.3 & $\mid-0.3$ & $\mid-0.3$ & $\mid-0.5$ & \begin{tabular}{|l|}
-1.2 \\
\end{tabular} & -3.9 & -7.7 & -36.8 \\
\hline & -3.5 & -1.9 & -1.0 & -0.7 & $|-0.4|$ & $|-0.3|$ & $|-0.3|$ & $-0.3 \mid$ & $|-0.4|$ & -0.7 & -1.2 & -1.6 & -3.1 \\
\hline & -34.1 & -9.2 & -2.4 & -1.2 & $|-0.4|$ & $|-0.3|$ & $|-0.3|$ & $|-0.3|$ & $|-0.5|$ & -1.1 & -3.6 & -6.7 & -23.2 \\
\hline & -3.9 & -2.0 & -1.0 & -0.7 & -0.4 & $|-0.3|$ & $|-0.3|$ & -0.3 & -0. & $\mid-0.6$ & -1.1 & -1.5 & -2.8 \\
\hline & -2.1 & -0.3 & -0.1 & 0.0 & 0.0 & 0.0 & 0.0 & 0.0 & 0.0 & 0.0 & 0.1 & 0.2 & 3.0 \\
\hline & 2.0 & 0.2 & 0.0 & 0.0 & 0.0 & 0.0 & 0.0 & 0.0 & 0.0 & 0.0 & -0.1 & -0.2 & -2.3 \\
\hline \multirow[t]{6}{*}{$1.5 \mathrm{Y}$} & -28.4 & -10.6 & -3.5 & -2.0 & -0.7 & $\mid$\begin{tabular}{|l|}
-0.4 \\
\end{tabular} & \begin{tabular}{|l|}
-0.4 \\
\end{tabular} & $\mid-0.5$ & $\mid-0.6$ & \begin{tabular}{|l|}
-1.6 \\
\end{tabular} & -5.6 & -12.1 & -50.0 \\
\hline & -4.7 & -2.7 & -1.5 & -1.1 & $|-0.6|$ & $|-0.4|$ & $|-0.4|$ & $-0.5 \mid$ & $-0.5 \mid$ & $\mid-0.9$ & -1.7 & -2.4 & -4.3 \\
\hline & -41.3 & -12.2 & -3.7 & -2.1 & $-0.7 \mid$ & $|-0.4|$ & $|-0.4|$ & $-0.5 \mid$ & $\mid-0.6$ & -1.5 & -5.1 & -10.2 & -29.7 \\
\hline & -5.3 & -2.9 & -1.6 & -1.2 & $|-0.6|$ & $|-0.4|$ & $|-0.4|$ & $|-0.5|$ & $|-0.5|$ & -0.9 & -1.6 & -2.2 & -3.8 \\
\hline & -2.5 & -0.4 & -0.1 & -0.1 & 0.0 & 0.0 & 0.0 & 0.0 & 0.0 & 0.0 & 0.1 & 0.4 & 4.4 \\
\hline & 2.5 & 0.3 & 0.0 & 0.0 & 0.0 & 0.0 & 0.0 & 0.0 & 0.0 & 0.0 & -0.1 & -0.4 & -3.3 \\
\hline \multirow[t]{6}{*}{$2 \mathrm{Y}$} & -36.5 & -14.5 & -5.3 & -1.9 & -0.8 & $\mid-0.6$ & $\mid-0.5$ & $\mid-0.6$ & \begin{tabular}{|l|}
-0.9 \\
\end{tabular} & -2.0 & -6.0 & -17.6 & -91.0 \\
\hline & -6.2 & -3.7 & -2.2 & -1.3 & -0.8 & $-0.6 \mid$ & $|-0.5|$ & -0.6 & $-0.8 \mid$ & -1.2 & -2.1 & -3.3 & -6.2 \\
\hline & -55.7 & -17.2 & -5.6 & -1.9 & -0.8 & $|-0.6|$ & $-0.5 \mid$ & -0.6 & $|-0.9|$ & -2.0 & -5.5 & -14.2 & -43.6 \\
\hline & -7.1 & -4.0 & -2.3 & -1.3 & $-0.8 \mid$ & -0.6 & $|-0.5|$ & -0.6 & $-0.8 \mid$ & -1.2 & -1.9 & -3.0 & -5.3 \\
\hline & -3.5 & -0.6 & -0.2 & -0.1 & 0.0 & 0.0 & 0.0 & 0.0 & 0.0 & 0.0 & 0.1 & 0.7 & 10.0 \\
\hline & 3.6 & 0.5 & 0.1 & 0.0 & 0.0 & 0.0 & 0.0 & 0.0 & 0.0 & -0.1 & -0.1 & -0.6 & -6.6 \\
\hline \multirow[t]{6}{*}{$3 \mathrm{Y}$} & -64.7 & -17.8 & $\mid-11.3$ & -2.9 & -1.4 & \begin{tabular}{l|l}
0.9 \\
\end{tabular} & 0.8 & $\mid-0.8$ & $\mid-1.3$ & -3.1 & -10.5 & -27.0 & -140.9 \\
\hline & 0.5 & -5.0 & -3.9 & -1.9 & -1.2 & -0.9 & $|-0.8|$ & -0.8 & $\mid-1.1$ & -1.8 & -3.2 & -4.9 & -8.8 \\
\hline & -122.7 & -21.1 & -12.6 & -3.0 & -1.4 & -0.9 & $|-0.8|$ & $-0.8 \mid$ & -1.2 & -3.0 & -9.3 & -20.7 & -57.5 \\
\hline & -12.6 & -5.6 & -4.3 & -2.0 & $-1.3 \mid$ & -0.9 & $|-0.8|$ & $-0.8 \mid$ & $\mid-1.1$ & -1.8 & -3.0 & -4.3 & -7.3 \\
\hline & -8.9 & -0.8 & -0.4 & -0.1 & $|-0.1|$ & 0.0 & 0.0 & 0.0 & 0.0 & 0.1 & 0.3 & 1.2 & 16.0 \\
\hline & 10.7 & 0.6 & 0.3 & 0.1 & 0.0 & 0.0 & 0.0 & 0.0 & $|-0.1|$ & -0.1 & -0.3 & -1.2 & -10.1 \\
\hline \multirow[t]{6}{*}{$5 \mathrm{Y}$} & -106.7 & -30.6 & $\mid-13.2$ & -5.9 & 2.2 & 1.5 & -1.2 & $\mid-1.3$ & $\mid-2.1$ & -4.8 & -17.3 & -45.5 & -471.9 \\
\hline & -18.1 & -8.6 & -5.5 & -3.6 & 2.0 & -1.5 & $\mid-1.2$ & -1.3 & -1.9 & -2.9 & -5.3 & -7.9 & -14 \\
\hline & -256.0 & -38.2 & -14.5 & -6.1 & -2.2 & -1.5 & $\mid-1.2$ & -1.3 & -2.1 & -4.6 & -14.9 & -32.2 & -88.5 \\
\hline & -23.2 & -10.0 & -6.1 & -3.8 & $|-2.1|$ & -1.5 & $\mid-1.2$ & $\mid-1.3$ & -1.8 & $|-2.7|$ & -4.7 & -6.7 & -11.5 \\
\hline & -18.8 & -1.6 & -0.5 & -0.3 & $|-0.1|$ & -0.1 & 0.0 & 0.0 & 0.1 & 0.1 & 0.5 & 2.4 & 38.9 \\
\hline & 23.1 & 1.3 & 0.3 & 0.2 & 0.1 & 0.0 & 0.0 & $-0.1 \mid$ & $-0.1 \mid$ & -0.2 & -0.5 & -2.4 & -20.9 \\
\hline \multirow[t]{6}{*}{$10 \mathrm{Y}$} & -172.3 & -69.5 & -30.2 & -10.0 & $\mid-4.4$ & -2.7 & $\mid-2.2$ & $\mid-2.4$ & -3.6 & $\mid-9.1$ & -34.6 & -103.0 & $\mathrm{ND}$ \\
\hline & -33.7 & -19.2 & -12.1 & -6.7 & -4.1 & -2.8 & -2.2 & -2.4 & -3.4 & $-5.6 \mid$ & -10.2 & -15.5 & -29.6 \\
\hline & -472.8 & -94.7 & -34.0 & -10.3 & $|-4.4|$ & -2.8 & $\mid-2.2$ & $-2.4 \mid$ & -3.6 & -8.7 & -28.2 & -60.5 & -159 \\
\hline & -47.5 & -24.3 & -14.3 & -7.3 & -4.2 & -2.8 & -2.2 & -2.4 & -3.3 & $-5.1 \mid$ & -8.7 & -12.3 & -20.9 \\
\hline & -33.9 & -5.0 & -1.1 & -0.5 & -0.3 & -0.2 & 0.0 & 0.1 & 0.2 & 0.3 & 1.1 & 6.7 & 146.5 \\
\hline & 27.4 & 2.5 & 0.6 & 0.4 & 0.2 & 0.1 & 0.0 & -0.1 & -0.2 & -0.3 & -1.3 & -7.0 & -58.7 \\
\hline
\end{tabular}

Table 1.4. CEV model $(\beta=0.8)$ : errors in bps on the implied volatility using the 6 approximations $\operatorname{ImpVol}(\operatorname{AppPrice}(2, \mathrm{~S} 0)), \operatorname{AppImpVol}(2, \mathrm{~S} 0)$, ImpVol (AppPrice $(2, K)), \quad \operatorname{AppImpVol}(2, \mathrm{~K}), \quad \operatorname{ImpVol}(\operatorname{AppPrice}(3, \mathrm{~S} 0))$ and ImpVol (AppPrice $(3, K)$ ). 


\begin{tabular}{|c|c|c|c|c|c|c|c|c|c|c|c|c|c|}
\hline \multirow[t]{6}{*}{$3 \mathrm{M}$} & 131.8 & -71.5 & -33.0 & -12.7 & -4.2 & -1.5 & -1.0 & -2.3 & -3.9 & -22.8 & -52.9 & -124.1 & -525.3 \\
\hline & -18.8 & -12.6 & -8.0 & -4.8 & -2.7 & -1.5 & -1.0 & -2.3 & -2.7 & -5.5 & -7.6 & -10.1 & -12.9 \\
\hline & ND & -134.0 & -43.9 & -14.2 & -4.3 & -1.6 & -1.0 & -2.3 & -3.7 & -18.5 & -34.8 & -57.1 & -84 \\
\hline & -24.4 & -15.4 & -9.3 & -5.3 & -2.8 & -1.5 & -1.0 & 2.2 & -2.7 & -4.9 & -6.5 & -8.4 & -10 \\
\hline & -31.8 & -9.5 & -2.1 & -0.5 & 0.2 & 0.1 & 0.0 & 0.9 & 0.5 & 0.6 & 3.4 & 12.8 & (1) \\
\hline & 57.2 & 11.7 & 2.1 & 0.5 & 0.2 & 0.1 & 0.0 & 1.0 & -0.7 & -1.1 & -3.3 & -9.1 & -20.3 \\
\hline \multirow[t]{6}{*}{$6 \mathrm{M}$} & 152.3 & -47.5 & 22.8 & 10.1 & -4.6 & -2.6 & \begin{tabular}{|l|}
-2.1 \\
\end{tabular} & 2.3 & -3. & -7.0 & -27.8 & -97.9 & $\overline{\mathrm{N}}$ \\
\hline & -28.2 & -14.0 & -9.4 & -6.1 & & -2.6 & -2.1 & -2.3 & & -4.4 & -8.2 & -13.4 & -23.2 \\
\hline & 56.9 & -63.4 & -26.3 & 10.8 & 1.7 & -2.6 & -2.1 & 2.3 & & -6.6 & -22.7 & .0 & 130 \\
\hline & -38.4 & -16.8 & -10.7 & -6.6 & & -8 & -2.1 & -2.3 & -3 & -4.1 & -7.1 & -10.9 & -17 \\
\hline & -31. & -3.3 & -1.1 & -0.6 & & & & 0 & & & 1.2 & 7 & \\
\hline & 41.7 & 3. & 1.2 & 0.6 & 0.3 & 0. & 0.0 & -0.1 & -0.2 & -0.3 & -1.1 & -6.7 & -41.0 \\
\hline \multirow[t]{6}{*}{$1 \mathrm{Y}$} & 257.5 & 05.8 & 34.8 & 18.9 & -6.5 & -4.8 & -4.2 & -4.3 & -7.4 & -18.9 & -77.7 & -203.6 & $\mathrm{~N}$ \\
\hline & -55.8 & 31.6 & 16.9 & 2.1 & 6.2 & -4.8 & 4.2 & 4.3 & 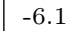 & -9.8 & 17.8 & -24.4 & -48. \\
\hline & ND & 164.6 & -40.5 & 20.7 & -6.7 & -4.8 & -4.2 & 4.3 & 7.1 & -16.9 & 52.8 & -90.9 & -227. \\
\hline & -84.9 & -41.8 & -19.7 & -13.4 & 6.3 & -4.8 & -4.2 & -4.2 & 5. & -8.7 & 14.3 & -18.3 & -30 \\
\hline & -63.4 & -11.2 & -2.4 & -1.5 & -0.6 & -0.3 & & 0. & . & 1.2 & 4.9 & 8.0 & 343 \\
\hline & 77.5 & 12.0 & 3.0 & 1.8 & 0.6 & .2 & -0.1 & 0.3 & -0.0 & -1.0 & -4.7 & -15.0 & 111 \\
\hline \multirow[t]{6}{*}{$1.5 \mathrm{Y}$} & 313.2 & 39.6 & -53.3 & 31.8 & 12.3 & -7.0 & & & & -25.4 & 118.7 & -574.7 & \\
\hline & & & -26.4 & 9.8 & & -7.0 & & 0.8 & & -13.7 & -25.7 & -36.7 & -60 \\
\hline & ND & 21.0 & 3.9 & 35.7 & & -7. & 6.2 & .8 & -8.4 & -22.4 & -73.0 & -131.2 & 27 \\
\hline & 24.7 & - & -32.0 & 22.6 & & & & 6.7 & 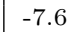 & -12.0 & -19.6 & .7 & 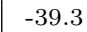 \\
\hline & -7 & 16.4 & -4.4 & -3.0 & & & & & & 2 & 0. & .6 & 470 . \\
\hline & 8 & 18.1 & 6 & 3 & & & & -0.7 & -0. & -1.6 & -8.1 & -29.8 & 150. \\
\hline \multirow[t]{6}{*}{$2 \mathrm{Y}$} & 395.3 & 187.6 & 79.0 & 31.6 & 4.5 & 11.0 & -8.3 & -8.7 & -12.8 & -33.0 & -125.5 & ND & $\mathrm{NI}$ \\
\hline & 04.9 & 3.9 & & 2.7 & & & .3 & 8.6 & & 17.8 & 30.4 & -49.9 & -94 . \\
\hline & ND & 4.7 & 8.3 & 4.7 & & & 3.3 & 8.6 & & & 78.5 & -172.3 & -342 \\
\hline & 30.4 & 3.1 & -48.5 & -25.5 & 4.1 & 1.0 & & 8.5 & & 15.2 & 22.8 & -32.7 & -50 \\
\hline & 5.8 & -25.5 & & -3.8 & -2.0 & & & & & 2.9 & ש & .9 & 770 \\
\hline & 99.9 & 28.0 & 10.5 & 4.6 & & & -0.2 & 1.0 & & -2.2 & -8.7 & 50.8 & -228.6 \\
\hline \multirow[t]{6}{*}{$3 \mathrm{Y}$} & 851.4 & 234.4 & -160.1 & 48.9 & 24.7 & -15.6 & -12.4 & 12.3 & & 51.2 & -279.0 & ND & $\mathrm{N}$ \\
\hline & & & & & & & & & & .3 & -47.8 & 73.4 & 3.3 \\
\hline & & & & & & & & & & 42.6 & & -231.1 & -397. \\
\hline & 75.3 & 3.1 & -1 & -41.3 & .0 & & & 2 & & -21.5 & -32.8 & .5 & -62 . \\
\hline & & .9 & -1 & -7.2 & -4.3 & & & & & 5. & 22.6 & 156.9 & 012. \\
\hline & . & .9 & & 8.8 & & & -0.5 & 1.5 & -2.5 & -3.7 & -21.6 & -89.8 & 295. \\
\hline \multirow[t]{6}{*}{$5 \mathrm{Y}$} & ND & 392.5 & 98.4 & 00.5 & 42.0 & 8.2 & 20.5 & -19.9 & -30.9 & -80.9 & $\mathrm{ND}$ & ND & $\mathrm{NI}$ \\
\hline & 20.6 & & 06.9 & -70.8 & & & & & & 40.9 & 76.7 & 17.4 & -219 \\
\hline & ND & & 7.5 & -113.0 & & & & & & -64.3 & -188.0 & -314.1 & -468. \\
\hline & 30.8 & -283.4 & -154.1 & 8.3 & 2.3 & -28.7 & 20.5 & 9.7 & .7 & -31.7 & -46.8 & -59.8 & -81 \\
\hline & 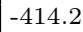 & 8.3 & -27.9 & -17.0 & -9.3 & -5 & 3 & 0 & & 9.4 & 49.9 & .0 & 498 \\
\hline & 0.0 & & & .7 & 7.9 & & -1.3 & -3.4 & -4.8 & -6.7 & -47.8 & -165.5 & 400. \\
\hline \multirow[t]{6}{*}{\begin{tabular}{|l|}
$10 Y$ \\
\end{tabular}} & ND & .7 & 6 & .2 & & .7 & 37.8 & 5.2 & -51.8 & -169.5 & ND & ND & $\mathrm{N}$ \\
\hline & & & & & & & & & & 6.8 & & -227.2 & \\
\hline & 45. & & & & & & & & & 8.9 & -304.8 & -431.9 & \\
\hline & & -7 & & & & & -37.8 & 35.2 & & -52.3 & & -85.6 & \\
\hline & 47.8 & & & & & & -2.6 & & & 19.8 & & 855.1 & דot. \\
\hline & $N$ & 411.2 & .9 & 4.6 & 23.9 & 1 & -2.6 & 7.4 & -9.8 & -15.8 & -135. & -323.5 & 406 \\
\hline
\end{tabular}

Table 1.5. CEV model $(\beta=0.2)$ : errors in bps on the implied volatility using the 6 approximations $\operatorname{ImpVol}(\operatorname{AppPrice}(2, \mathrm{~S} 0)), \operatorname{AppImpVol}(2, \mathrm{~S} 0)$, $\operatorname{ImpVol}(\operatorname{AppPrice}(2, \mathrm{~K})), \quad \operatorname{AppImpVol}(2, \mathrm{~K}), \quad \operatorname{ImpVol}(\operatorname{AppPrice}(3, \mathrm{~S} 0))$ and ImpVol (AppPrice $(3, K))$. 


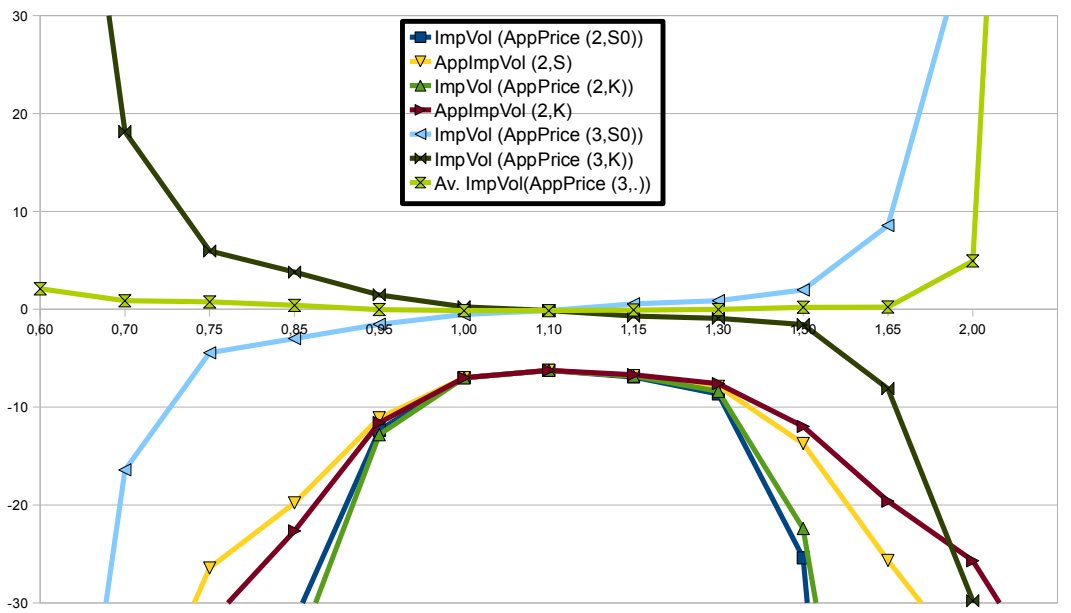

Fig. 1.2. CEV model $(\beta=0.2)$ : errors in bps on the implied volatility using the 7 approximations $\operatorname{ImpVol}(\operatorname{App} \operatorname{Price}(2, \mathrm{~S} 0)), \operatorname{AppImpVol}(2, \mathrm{~S} 0)$, ImpVol (AppPrice $(2, \mathrm{~K})), \quad \operatorname{AppImpVol}(2, \mathrm{~K}), \quad \operatorname{ImpVol}(\operatorname{AppPrice}(3, \mathrm{~S} 0))$, ImpVol (AppPrice $(3, K))$ and Av. ImpVol (AppPrice $(3,)$.

\begin{tabular}{|c||r|r|r|r|r|r|r|r|r|r|r|r|r|}
\hline $3 \mathrm{M}$ & -0.41 & -0.13 & -0.04 & -0.02 & -0.02 & -0.01 & 0.00 & 0.00 & 0.00 & 0.00 & 0.01 & 0.00 & -0.01 \\
\hline $6 \mathrm{M}$ & -0.16 & -0.04 & -0.03 & -0.02 & -0.01 & -0.01 & 0.00 & -0.01 & 0.00 & -0.01 & 0.00 & 0.01 & 0.04 \\
\hline $1 \mathrm{Y}$ & -0.05 & -0.06 & -0.04 & -0.03 & -0.01 & -0.02 & -0.01 & -0.01 & 0.00 & -0.01 & 0.00 & 0.00 & 0.34 \\
\hline $1.5 \mathrm{Y}$ & -0.02 & -0.07 & -0.04 & -0.03 & -0.02 & -0.02 & -0.01 & -0.01 & -0.01 & -0.01 & 0.00 & 0.01 & 0.54 \\
\hline $2 \mathrm{Y}$ & 0.06 & -0.08 & -0.05 & -0.03 & -0.02 & -0.02 & -0.02 & -0.01 & -0.01 & -0.01 & -0.01 & 0.01 & 1.69 \\
\hline $3 \mathrm{Y}$ & 0.89 & -0.09 & -0.06 & -0.03 & -0.02 & -0.02 & -0.02 & -0.02 & -0.01 & -0.01 & -0.01 & 0.02 & 2.98 \\
\hline $5 \mathrm{Y}$ & 2.17 & -0.16 & -0.06 & -0.04 & -0.03 & -0.03 & -0.03 & -0.02 & -0.01 & -0.01 & -0.01 & 0.01 & 8.99 \\
\hline $10 \mathrm{Y}$ & -3.24 & -1.24 & -0.23 & -0.05 & -0.05 & -0.05 & -0.04 & -0.03 & -0.02 & -0.01 & -0.12 & -0.13 & 43.89 \\
\hline
\end{tabular}

Table 1.6. CEV model $(\beta=0.8)$ : errors in bps on the implied volatility using Av . ImpVol (AppPrice $(3,$.$) )$

\begin{tabular}{|c||r|r|r|r|r|r|r|r|r|r|r|r|r|}
\hline $3 \mathrm{M}$ & 12.69 & 1.08 & 0.01 & 0.00 & -0.01 & -0.01 & 0.00 & -0.95 & -0.60 & -0.27 & 0.01 & 1.86 & 10.08 \\
\hline $6 \mathrm{M}$ & 5.36 & 0.07 & 0.07 & 0.03 & 0.00 & -0.02 & -0.02 & -0.02 & 0.00 & 0.02 & 0.04 & 0.54 & 26.98 \\
\hline $1 \mathrm{Y}$ & 7.01 & 0.37 & 0.31 & 0.14 & -0.04 & -0.07 & -0.06 & -0.06 & 0.01 & 0.11 & 0.13 & 1.52 & 116.22 \\
\hline $1.5 \mathrm{Y}$ & 2.09 & 0.87 & 0.76 & 0.40 & -0.03 & -0.14 & -0.14 & -0.07 & -0.02 & 0.21 & 0.23 & 4.94 & 159.77 \\
\hline $2 \mathrm{Y}$ & -2.93 & 1.24 & 1.46 & 0.40 & -0.12 & -0.21 & -0.23 & -0.14 & 0.02 & 0.34 & 0.28 & 13.05 & 270.84 \\
\hline $3 \mathrm{Y}$ & -49.57 & 2.02 & 3.24 & 0.82 & -0.19 & -0.50 & -0.50 & -0.34 & 0.05 & 0.69 & 0.49 & 33.54 & 358.51 \\
\hline $5 \mathrm{Y}$ & -540.41 & -13.94 & 3.99 & 2.33 & -0.69 & -1.31 & -1.27 & -0.77 & 0.22 & 1.37 & 1.04 & 87.77 & 549.35 \\
\hline $10 \mathrm{Y}$ & $\mathrm{ND}$ & -739.31 & -75.21 & 8.58 & 3.84 & -0.94 & -2.64 & -1.96 & 0.38 & 2.00 & 7.99 & 265.78 & 995.47 \\
\hline
\end{tabular}

Table 1.7. CEV model $(\beta=0.2)$ : errors in bps on the implied volatility using Av . ImpVol (AppPrice $(3,)$. 